\title{
Growing and moving low-mass planets in non-isothermal disks
}

\author{
S.-J. Paardekooper ${ }^{1,2}$ and G. Mellema ${ }^{3,2}$ \\ 1 Department of Applied Mathematics and Theoretical Physics, University of Cambridge, Wilberforce Road, Cambridge CB3 0WA, \\ UK \\ e-mail: S.Paardekooper@damtp.cam.ac.uk \\ 2 Leiden Observatory, Leiden University, Postbus 9513, 2300 RA Leiden, The Netherlands \\ 3 Stockholm Observatory, AlbaNova University Center, Stockholm University, 10691 Stockholm, Sweden \\ e-mail: garrelt@astro.su.se
}

Received 31 August 2007 / Accepted 21 November 2007

\begin{abstract}
Aims. We study the interaction of a low-mass planet with a protoplanetary disk with a realistic treatment of the energy balance by doing radiation-hydrodynamical simulations. We look at accretion and migration rates and compare them to isothermal studies. Methods. We used a three-dimensional version of the hydrodynamical method RODEO, together with radiative transport in the fluxlimited diffusion approach.

Results. The accretion rate, as well as the torque on the planet, depend critically on the ability of the disk to cool efficiently. For densities appropriate to $5 \mathrm{AU}$ in the solar nebula, the accretion rate drops by more than an order of magnitude compared to isothermal models, while at the same time the torque on the planet is positive, indicating outward migration. It is necessary to lower the density by a factor of 2 to recover inward migration and more than 2 orders of magnitude to recover the usual type I migration. The torque appears to be proportional to the radial entropy gradient in the unperturbed disk. These findings are critical for the survival of protoplanets, and they should ultimately find their way into population synthesis models.
\end{abstract}

Key words. hydrodynamics - methods: numerical - stars: planetary systems

\section{Introduction}

Planet formation takes place in protoplanetary disks that are commonly found around young stars (Beckwith \& Sargent 1996). These disks play a critical role in structuring the forming planetary system in terms of masses and orbits, and planet-disk interaction is therefore a very important process to study in order to understand planet formation.

Planet-disk interaction affects a forming planet in three ways. First of all, the mass that the disk is able to supply to the young planet is limited, which leads to a maximum gas mass that a planet can achieve (Lubow et al. 1999; Kley 1999; D'Angelo et al. 2003b; Bate et al. 2003). This limiting mass is of the order of a few Jupiter masses $\left(M_{\mathrm{J}}\right)$. But also for planets of lower mass the disk regulates the mass accretion, which becomes especially apparent in three-dimensional numerical simulations (D'Angelo et al. 2003b).

Second, the disk is able to change the orbital radius of the planet through tidal interaction (Goldreich \& Tremaine 1980). Depending on the mass of the planet, two modes of migration can be distinguished (Ward 1997): type I migration for lowmass planets that generate a linear disk response, and type II for high-mass, gap-opening planets. Recently a third type of migration was put forward, in which strong corotational torques force a very fast mode of migration (Masset \& Papaloizou 2003; Artymowicz 2004). This type III migration is very sensitive to local density gradients, in terms of migration speed and even migration direction, and therefore the structure of the gas disk determines the outcome of the migration process. This is unlike type I and type II migration, for which all planets move inward and only the time scale varies with disk properties.
Finally, the eccentricity of the planet's orbit may be affected by the disk. Usually, eccentricity is damped (Artymowicz 1993), but high-mass planets $\left(M_{\mathrm{p}}>5 M_{\mathrm{J}}\right)$ that open deep gaps may experience eccentricity growth (Papaloizou et al. 2001; Kley \& Dirksen 2006). However, due to possible saturation of corotation resonances planets of $M_{\mathrm{p}} \approx M_{\mathrm{J}}$ may pick up significant eccentricity during the gap formation process (Sari \& Goldreich 2004), although this requires a slightly eccentric orbit to start with (Goldreich \& Sari 2003).

Observations of the masses and orbits of extrasolar planets provide insight in the result of planet-disk interaction. One of the most striking results that were obtained is the discovery of a new class of planets, the so-called Hot Jupiters. They are gas giant planets orbiting very close to their central star. It is unlikely that they have formed in situ (Papaloizou \& Terquem 1999) and inward migration through planet-disk tidal interaction has been put forward as the explanation for their existence.

The main problem for this scenario is that the time scales for migration as obtained from analytical (Tanaka et al. 2002) and numerical (Bate et al. 2003; D'Angelo et al. 2003b) arguments are usually smaller than or comparable to the total lifetime of the disk. This means that theory essentially predicts that all planets should fall onto the central star, which poses a problem for planet formation theory. If it is already hard to form a single giant planet within the lifetime of the disk (Pollack et al. 1996), how are we going to form a huge amount of them, of which only a tiny fraction survives? Clearly a stopping mechanism is required that prevents planets from migrating all the way towards the star.

One possibility is that the planet encounters an inner hole in the disk, possibly created by the magnetic field of the central star. When there is no disk around to interact with, the planet will 
stop migrating. Another possibility is that the planet undergoes type III outward migration at some point (Masset \& Papaloizou 2003). Finally, direct interaction of the disk with a magnetic field may stop the planet, either by a toroidal magnetic field (Terquem 2003) or, for a low-mass planet, its migration may be slowed down through magnetic turbulence (Nelson \& Papaloizou 2004).

However, most numerical hydrodynamical work on planetdisk interaction lacks proper treatment of the energy balance, with only a few notable exceptions (D'Angelo et al. 2003a; Klahr \& Kley 2006; Morohoshi \& Tanaka 2003). The usually adopted locally isothermal equation of state assumes that all excess energy generated by compression, viscous dissipation or shocks can be radiated away efficiently, thereby keeping the temperature profile fixed. However, the ability of the disk to cool is strongly linked to the opacity, and therefore to the density.

The effects of temperature structure on planet migration in realistic disks were studied by Jang-Condell \& Sasselov (2003, 2004,2005 ) who used a detailed disk model including radiative transfer to calculate the torque on the planet in an analytical way. They found that temperature perturbations resulting from shadowing and illumination at the surface of the disk can decrease the migration rate by a factor of 2. Menou \& Goodman (2004) also find reduced migration rates in realistic T Tauri $\alpha$-disks, and they point out the importance of sudden changes in the opacity, for example near the snow line.

In this study, we aim at relaxing the isothermal assumption in hydrodynamical simulations of planet-disk interaction. The first results on planet migration were presented in Paardekooper \& Mellema (2006a), where it was shown that low-mass planets may migrate outward in disks with realistic temperature structures. In this paper, we continue our study of migration of lowmass objects, with more eye for detail than in Paardekooper \& Mellema (2006a), and include results on accretion.

In contrast with Klahr \& Kley (2006), we focus on low-mass planets that do not open a gap in the disk. For these deeply embedded planets temperature effects are the most important. However, due to their low mass their gravitational sphere of influence is small, and one needs very high resolution locally to resolve the atmosphere of the planet. Furthermore, because radiation is the main cooling agent, we need to perform radiationhydrodynamical simulations. This is a major leap from isothermal simulations, because we are not only adding an equation for the gas energy but also the evolution of the radiation field needs to be followed.

The plan of this paper is as follows. We briefly review the relevant cooling time scale in Sect. 2, effects of radiation in Sect. 3 and the condition for convection in Sect. 4 . In Sect. 5 we present the adopted disk model, and in Sect. 6 we discuss the numerical method. In Sect. 7 we present the results, from simple isothermal models to the full radiation-hydrodynamical models. We give a discussion on the results in Sect. 8, and we conclude in Sect. 9.

\section{Cooling properties}

We take the analytical opacity data from Bell \& Lin (1994), which state that for low temperatures (beyond the snow line) the Rosseland mean opacity in $\mathrm{cm}^{-1}$ is:

$\kappa=\kappa_{0} \rho T^{2}$,

where $\rho$ is the density in $\mathrm{g} \mathrm{cm}^{-3}, T$ denotes the temperature (K) and $\kappa_{0}=2.0 \times 10^{-4}$ is a constant. We discuss the cooling properties of the disk as a function of opacity, starting with the optically thin regime.
The time scale for the coupling between gas and radiation is given by

$t_{\text {coupling }}=\frac{1}{\kappa c}$

where $c$ is the speed of light in vacuum. When $t_{\text {coupling }}$ is larger than the dynamical time scale $t_{\text {dyn }}$, which equals the orbital time scale of the planet, the gas can not transfer its internal energy to the radiation field and therefore the gas will not cool efficiently through radiation. Plugging in the opacity of Eq. (1), and assum$\operatorname{ing} T \approx 50 \mathrm{~K}$, which is appropriate for the location of Jupiter in the solar nebula, we find that the density near the planet should satisfy

$\rho>10^{-18} \mathrm{~g} \mathrm{~cm}^{-3}$,

in order to cool through radiation. At the midplane of a protoplanetary disk in the planet forming region this condition is always satisfied.

In general, when a region is optically thin and Eq. (3) holds it can cool efficiently because all its thermally emitted photons can escape from this region. In a protoplanetary disk the scale length of interest is the disk thickness $H$. For planet-disk interaction specifically, most of the torque on the planet comes from material that is closer than $\sim 2 H$ from the planet (Bate et al. 2003). The condition for the disk to be optically thin over one pressure scale height is given by:

$\kappa H<1$.

Putting in our opacity law Eq. (1) we can write a condition for the density:

$\rho<\frac{1}{\kappa_{0} T^{2} H}$

At the location of Jupiter in a typical protoplanetary disk $T \approx$ $50 \mathrm{~K}$ and $h \equiv H / r=0.05$, where $r$ is the orbital distance of Jupiter. Putting in these values, we find that for the disk to be optically thin over one pressure scale height $\rho<5.1 \times$ $10^{-13} \mathrm{~g} \mathrm{~cm}^{-3}$, while the density at $5 \mathrm{AU}$ in the minimum mass solar nebula (MMSN) is given by $\rho=10^{-11} \mathrm{~g} \mathrm{~cm}^{-3}$.

When a region is optically thick, it can still cool efficiently depending on the local conditions. Consider a sphere of radius $H$ around the position of the planet. The flux through this sphere in the optically thick case can be approximated by

$F_{\mathrm{R}}=\frac{\sigma T^{4}}{\tau}$

where $\tau=\kappa H$ is the optical depth over the radius of the sphere and $\sigma$ is the Stefan-Boltzmann constant. The internal energy density in the sphere is given by:

$\epsilon=\frac{p}{\Gamma-1}=\frac{\rho \frac{R}{\mu} T}{\Gamma-1}=\frac{\tau \frac{R}{\mu}}{\kappa_{0} T H(\Gamma-1)}$,

where $p$ denotes gas pressure, $\Gamma$ is the adiabatic exponent, $R$ is the universal gas constant and $\mu$ is the mean molecular weight. The cooling time scale is given by the total internal energy content of the sphere divided by the total energy that flows through the surface of the sphere:

$t_{\text {cool }}=\frac{\epsilon \frac{4}{3} \pi H^{3}}{F_{\mathrm{R}} 4 \pi H^{2}}=\frac{\epsilon H}{3 F_{\mathrm{R}}}=\frac{\tau^{2} \frac{R}{\mu}}{3(\Gamma-1) \kappa_{0} \sigma T^{5}}$. 
Putting in the density and temperature appropriate for the location of Jupiter $\left(\rho=10^{-11} \mathrm{~g} \mathrm{~cm}^{-3}, T=50 \mathrm{~K}\right.$ and $\left.\mu=2.4\right)$ we find that $t_{\mathrm{cool}} \approx 10 t_{\mathrm{dyn}}$, where the dynamical time scale $t_{\mathrm{dyn}}$ equals the orbital time scale of the planet. Cooling is only efficient when $t_{\text {cool }}<t_{\text {dyn }}$, and therefore we conclude that cooling is not efficient for these parameters.

It is easy to show that the cooling time scale depends on the distance to the central star through:

$t_{\text {cool }} \propto \frac{\rho^{2}(r) H^{2}(r)}{T(r)}$.

When we assume a constant aspect ratio $h$, which implies that the temperature varies with radius as $r^{-1}$, and a power law for the density with index $-3 / 2$ we see that the cooling time does not depend on radius. This means that

$\frac{t_{\mathrm{cool}}}{t_{\mathrm{dyn}}} \approx 10\left(\frac{r}{5 \mathrm{AU}}\right)^{-\frac{3}{2}}$.

This means that at approximately $15 \mathrm{AU}$ in the MMSN $t_{\text {cool }} \approx$ $t_{\text {dyn }}$. Inside this radius, cooling will not be efficient.

Both Eqs. (5) and (8) have a strong temperature dependence, and therefore it is not possible to determine in advance if a disk with an embedded planet that changes the local temperature and density will be able to cool efficiently. However, this analysis shows that for typical densities and temperatures including proper cooling mechanisms is essential for planet-disk interaction.

Summarizing, for a fixed temperature of $50 \mathrm{~K}$ at $5 \mathrm{AU}$, we can distinguish four cooling regimes:

- For the lowest densities (see Eq. (3)) the gas can not cool through radiation.

- For somewhat higher densities, gas is able to cool through radiation but the disk is still optically thin over a distance $H$.

- Even higher densities make the disk optically thick over $H$, which means that thermally emitted photons do not immediately leave the region that provides the torque on the planet.

- For the highest densities the disk is optically thick and $t_{\text {cool }}>$ $t_{\text {dyn }}$, which means that the disk can not cool efficiently.

Note that only the second regime resembles the isothermal limit, while the first regime is not important for low-mass planets that remain deeply embedded. In the third and the fourth regime, temperature effects may play a large role in determining the total torque onto the planet.

\section{Radiative effects}

In general, radiation is not only a cooling agent but it may also dynamically change the velocity structure around the planet. The effect of radiation on the gas velocity goes through radiation pressure, which, in the isotropic case, is given by

$P=\frac{1}{3} a T^{4}$,

where $a$ is the radiation constant. The gas pressure $p$ is again given by the ideal gas law, and we can then write:

$\frac{P}{p}=\frac{\frac{1}{3} a T^{4}}{\rho \frac{R}{\mu} T}=\frac{\mu a T^{3}}{3 \rho R}$.

For the typical temperature at $5 \mathrm{AU}, T=50 \mathrm{~K}$, we get:

$\frac{P}{p}=\frac{1.0 \times 10^{-17}}{\rho}$.
For a typical midplane density of $\rho=10^{-11} \mathrm{~g} \mathrm{~cm}^{-3}$ radiation pressure is negligible compared to gas pressure.

This is an important result, because it shows that near a deeply embedded planet the dynamical effects of radiation are not important. This means that the gas pressure and velocity structure will be almost the same as in the locally isothermal case. This greatly simplifies the torque analysis: in regions where the temperature rises because of gas compression or the release of potential energy the density must be lower than in the isothermal case to arrive at the same pressure. Therefore these regions will exert a lower torque onto the planet. Any asymmetry in heating will therefore show up as a torque asymmetry, which may alter the total torque balance on the planet considerably.

\section{Convection}

Heat transport in a planetary envelope can occur through radiation or through convection. Convection sets in when the temperature gradient becomes too steep for radiation to transport energy towards the surface of the planet. As is well known from theory of stellar interiors, this happens when

$\frac{\partial T}{\partial z}<\left.\frac{\partial T}{\partial z}\right|_{\mathrm{ad}}$,

where $z$ is the direction towards the surface of the planet and the right hand side denotes the adiabatic temperature gradient, which can be expressed as:

$\left.\frac{\partial T}{\partial z}\right|_{\mathrm{ad}}=-\frac{T}{H} \frac{\Gamma}{\Gamma-1}$.

We can make a simple estimate of the importance of convective energy transport by approximating the actual temperature gradient in the planetary envelope as:

$\frac{\partial T}{\partial z} \approx \frac{T_{\mathrm{neb}}-T_{\mathrm{c}}}{R_{\mathrm{R}}}$

where $T_{\text {neb }}$ is the temperature of the surrounding nebula, $T_{\mathrm{c}}$ is the central temperature of the planet and $R_{\mathrm{R}}$ denotes the Roche lobe of the planet. The assumption is that the planet connects to the disk at a distance given by the Roche lobe, and that the disk remains unperturbed from this distance. This is the usual assumption in one-dimensional planet formation models (Pollack et al. 1996).

When we further assume that the low-mass planets do not change to local pressure scale height in the disk, we can plug Eq. (15) and (16) into Eq. (14) to obtain a maximum value for the central temperature of the planet:

$T_{\mathrm{c}}<\left(1+\frac{R_{\mathrm{R}}}{H} \frac{\Gamma}{\Gamma-1}\right) T_{\text {neb }}$

For a $5 M_{\oplus}$ planet embedded in a disk with aspect ratio $h=0.05$ and adiabatic exponent $\Gamma=1.4$ we find that $T_{\mathrm{c}}<2.21 T_{\text {neb }}$.

As will become apparent in Sect. 7 , the direction of migration critically depends on the radial entropy gradient in the disk, or, in other words, whether Eq. (14) is satisfied in the radial direction. For a disk that follows a power law in temperature and density with indices $\beta$ and $\alpha$, respectively, the entropy also follows a power law with index

$\mathcal{S}=\beta-(\Gamma-1) \alpha$.

Our nominal model, with $\beta=-1$ and $\alpha=-3 / 2$, has a negative entropy gradient. Note that because of the rotation of the disk, this does not lead to convection. Also, we did not find evidence for an instability due to baroclinic effects (Klahr \& Bodenheimer 2003). 


\section{Model design}

Our model consists of three objects: the central star, the planet and the disk. Below, we give a short description of each of these components. Throughout this paper, we will work in a spherical polar coordinate system $(r, \theta, \phi)$ that co-rotates with the planet.

\subsection{Central star}

The central star is the main source of gravity and is located in the origin of our coordinate system. This makes the system noninertial, the principle upon which the radial velocity searches for extrasolar planets are based. Although we take the acceleration of the coordinate frame into account, for the low-mass planets the effect is negligible. We take the mass of the star to be $1 M_{\odot}$.

\subsection{Planet}

Our coordinate frame rotates with the angular velocity of the planet, and because we keep the planet on a fixed circular orbit it resides at a fixed location on the grid, $(r, \theta, \phi)=\left(r_{\mathrm{p}}, \pi / 2, \pi\right)$. The potential of the planet is smoothed over 2 grid cells on the highest level of refinement, which is always much smaller than the Roche lobe of the planet (see Sect. 6). This way, we always resolve the potential. The planet is able to accrete matter from the disk without changing its dynamical mass. We vary the mass of the planet between 0.6 and $300 M_{\oplus}$ for the isothermal models, which spans the whole range from the linear regime of type I migration to a gap-opening Jupiter-mass planet. For the radiationhydrodynamical models we focus on a planet of $5 M_{\oplus}$, which is well inside the linear regime (see however Masset et al. 2006).

\subsection{Disk}

The disk is three-dimensional, extending from $r=0.4 r_{\mathrm{p}}$ to $r=$ $2.5 r_{\mathrm{p}}$ in the radial direction ( $r_{\mathrm{p}}$ is the distance from the central star to the planet). In the azimuthal direction the computational domain is bounded by $0 \leq \phi \leq 2 \pi$, while in the polar direction we go up to 2.5 pressure scale heights above the midplane of the disk: $\pi / 2-5 H / 2 r<\theta<\pi / 2$.

\subsubsection{Gas}

The scale height $H$ varies linearly with radius initially, with $h=H / r=0.05$. The initial temperature profile is found from assuming hydrostatic equilibrium in the vertical direction and therefore matches the temperature profile of the isothermal simulations. The density is also a power law initially, with index $-3 / 2$. We vary the midplane density at the location of the planet to investigate different cooling regimes. Our nominal value is $10^{-11} \mathrm{~g} \mathrm{~cm}^{-3}$, which is appropriate for the location of Jupiter in the minimum mass solar nebula. The initial velocities are zero in the radial and polar direction, and the Keplerian speed in the azimuthal direction, with a correction for the radial pressure gradient. The equation of state is given by the ideal gas law.

We model the disk as an ideal, inviscid fluid, and therefore its evolution is governed by the Euler equations. These can be cast in the following form:

$\frac{\partial \boldsymbol{W}}{\partial t}+\frac{\partial \boldsymbol{F}_{r}}{\partial r}+\frac{\partial \boldsymbol{F}_{\theta}}{\partial \theta}+\frac{\partial \boldsymbol{F}_{\phi}}{\partial \phi}=\boldsymbol{S}$, in which $\boldsymbol{W}$ is the state vector, $\boldsymbol{F}$ are the fluxes in the three coordinate directions and $\boldsymbol{S}$ is the source vector. The state consists of the conserved quantities:

$\boldsymbol{W}=r^{2} \sin \theta\left(\rho, \rho v_{r}, \rho v_{\theta}, \rho v_{\phi}, e\right)^{\mathrm{T}}$,

where $\rho$ is the density, $v_{r}$ is the radial velocity, $v_{\theta}$ and $v_{\phi}$ are the two angular velocities and $e$ is the total energy density:

$e=\frac{1}{2} \rho\left(v_{r}^{2}+r^{2} v_{\theta}^{2}+r^{2} \sin ^{2} \theta\left(v_{\phi}+\Omega\right)^{2}\right)-\rho \Phi+\frac{p}{\Gamma-1}$.

Here $\Omega$ is the angular velocity of the coordinate frame, $\Phi$ denotes the potential, $p$ is the pressure and $\Gamma$ is the adiabatic exponent. The three fluxes are given by:

$$
\begin{aligned}
& \boldsymbol{F}_{r}=r^{2} \sin \theta\left(\rho v_{r}, \rho v_{r}^{2}+p, \rho v_{r} v_{\theta}, \rho v_{r} v_{\phi}, \rho v_{r} w\right)^{\mathrm{T}} \\
& \boldsymbol{F}_{\theta}=r^{2} \sin \theta\left(\rho v_{\theta}, \rho v_{r} v_{\theta}, \rho v_{\theta}^{2}+p / r^{2}, \rho v_{\theta} v_{\phi}, \rho v_{\theta} w\right)^{\mathrm{T}} \\
& \boldsymbol{F}_{\phi}=r^{2} \sin \theta\left(\rho v_{\phi}, \rho v_{r} v_{\phi}, \rho v_{\theta} v_{\phi}, \rho v_{\phi}^{2}+\frac{p}{r^{2} \sin ^{2} \theta}, \rho v_{\phi} w\right)^{\mathrm{T}},
\end{aligned}
$$

where $w$ is the enthalpy of the fluid:

$w=\frac{e+p}{\rho}$

The source vector $S$ is then:

$$
\begin{aligned}
& \boldsymbol{S}=r^{2} \sin \theta \\
& \times\left(\begin{array}{c}
0 \\
\rho r \sin ^{2} \theta\left(v_{\phi}+\Omega\right)^{2}-\rho \frac{\partial \Phi}{\partial r}+\frac{2 p}{r} \\
-2 \rho \frac{v_{r}}{r} v_{\theta}+\sin \theta \cos \theta \rho\left(v_{\phi}+\Omega\right)^{2}-\frac{\rho}{r^{2}} \frac{\partial \Phi}{\partial \theta}+\frac{\cot \theta p}{r^{2}} \\
-2 \rho \frac{v_{r}}{r}\left(\Omega+v_{\phi}\right)-2 \cot \theta \rho v_{\theta}\left(\Omega+v_{\phi}\right)-\frac{\rho}{r^{2} \sin ^{2} \theta} \frac{\partial \Phi}{\partial \phi} \\
-2 \rho\left(v_{r} \frac{\partial \Phi}{\partial r}+v_{\theta} \frac{\partial \Phi}{\partial \theta}+v_{\phi} \frac{\partial \Phi}{\partial \phi}\right)-\rho \Omega \frac{\partial \Phi}{\partial \phi}
\end{array}\right) .
\end{aligned}
$$

The reason for not including an anomalous turbulent viscosity as in Bate et al. (2003) and D'Angelo et al. (2003b) is that the usual $\alpha$-prescription (Shakura \& Sunyaev 1973) is not a good description of the magnetic turbulence that is probably the source of viscosity (Balbus \& Hawley 1990). The turbulent nature of the disk leads to large temporal and spatial variations in $\alpha$ (Papaloizou \& Nelson 2003). Neglecting viscosity altogether means that our results are valid in regions of the disk where the ionization fraction is not high enough to sustain magnetic turbulence.

\subsubsection{Radiation}

Planets that do not open gaps $\left(M_{\mathrm{p}} \ll M_{\mathrm{J}}\right)$ are deeply embedded in the disk where the material is very optically thick. It is therefore appropriate to consider radiative transfer in the diffusion limit. However, because the density drops more than an order of magnitude over a few pressure scale heights above the midplane it is necessary to deal with the optically thin regime as well.

We start by considering a medium at rest, i.e. we set the velocity of the gas to zero. In the diffusion limit there is essentially no angular information in the radiation field, which is then governed by a diffusion equation for its energy density $E$ :

$\frac{\partial E}{\partial t}-\nabla \cdot \frac{c}{3 \kappa} \nabla E=\kappa c(B-E)$

where $c$ denotes the speed of light, $\kappa$ is the opacity in $\mathrm{cm}^{-1}$ and $B$ is the radiative source term. We consider only thermal emission from the disk, therefore

$B=a T^{4}$, 
where $a$ is the radiation constant and $T$ is the gas temperature. In order to conserve total energy an extra source term $S_{\text {rad }}=$ $-\kappa c(B-E)$ appears in the gas energy equation. We work with a Rosseland mean opacity that is typical for the MMSN (see Bell $\&$ Lin 1994). Initially we set $E=B$ in all simulations.

In the optically thin streaming limit the evolution of the radiation energy is given by:

$\frac{\partial E}{\partial t}+\nabla \cdot(c E \hat{\boldsymbol{n}})=\kappa c(B-E)$,

where $\hat{\boldsymbol{n}}$ is a unit vector in the direction of propagation of the light front. Note first of all that this is an advection equation rather than a diffusion equation, and second that there is a strong angular dependence of the radiation field. Naively applying Eq. (27) in the optically thin case will lead to large errors.

However, because the most important part of the disk is optically thick it is still useful to start with Eq. (27) but to avoid divergences in the optically thin case. Note that avoiding divergences is different from obtaining the correct solution, which would require a method based on discrete ordinates or on a Monte Carlo algorithm. Both of these methods have great difficulties with the case of a very optically thick disk.

We write Eqs. (27) and (29) in the more general form:

$\frac{\partial E}{\partial t}+\nabla \cdot \boldsymbol{F}_{\mathrm{R}}=\kappa c(B-E)$,

in which the radiative flux $\boldsymbol{F}_{\mathrm{R}}$ is given by

$\boldsymbol{F}_{\mathrm{R}}=-\frac{c \lambda}{\kappa} \nabla E$

where $\lambda$ is called the flux limiter. The definition of $\boldsymbol{F}_{\mathrm{R}}$ in Eq. (31) in terms of $E$ removes the need for solving a differential equation for $\boldsymbol{F}_{\mathrm{R}}$. Closing the radiative moment equations this way is called flux-limited diffusion (FLD) (Levermore \& Pomraning 1981). The flux-limiter $\lambda$ deals with the transition from the diffusion limit to the streaming limit. The exact functional form of $\lambda$ also implicitly determines the angular dependence of the radiation field. For an overview of different forms see Kley (1989). We have used

$\lambda= \begin{cases}\frac{2}{3+\sqrt{9+10 R^{2}}} & \text { for } 0 \leq R \leq 2 ; \\ \frac{10}{10 R+9+\sqrt{180 R+81}} & \text { for } R>2,\end{cases}$

where

$R=\frac{1}{\kappa} \frac{|\nabla E|}{E}$

but the results do not depend sensitively on the adopted form of $\lambda$, mainly because of the high optical depth of the disk near the planet.

Flux-limited diffusion typically gives poor results in the streaming limit (Hayes \& Norman 2003), but for our case of a deeply embedded planet it is a reliable and relatively cheap method for doing radiative transfer.

When the velocity of the gas is taken into account Eq. (30) needs to be extended in two ways to account for advection of radiative energy and momentum transfer between gas and radiation. The full equation for the evolution of the radiative energy then reads:

$\frac{\partial E}{\partial t}+\nabla \cdot\left(\boldsymbol{F}_{\mathrm{R}}+\boldsymbol{v} E+\boldsymbol{v} \cdot \mathcal{P}\right)=\kappa c(B-E)-\frac{\kappa}{c} \boldsymbol{v} \cdot \boldsymbol{F}_{\mathrm{R}}$,

where $\mathcal{P}$ is the radiative stress tensor, given by

$\mathcal{P}=E \mathcal{T}_{\text {Edd }}$.
The Eddington tensor $\mathcal{T}_{\text {Edd }}$ is calculated using

$\mathcal{T}_{\text {Edd }}=\frac{1}{2}\left[\left(1-f_{\text {Edd }}\right) \mathcal{I}+\left(3 f_{\text {Edd }}-1\right) \hat{n} \hat{n}\right]$,

where $\hat{\boldsymbol{n}}=\nabla E /|\nabla E|$. The Eddington factor $f_{\text {Edd }}$ can be calculated from the streaming factor $f_{\mathrm{s}}=\left|\boldsymbol{F}_{\mathrm{R}}\right| / c E$ :

$f_{\mathrm{Edd}}= \begin{cases}\frac{1}{3}+\frac{1}{6} f_{\mathrm{s}}^{2} & \text { for } 0 \leq f_{\mathrm{s}} \leq 0.4 \\ \frac{5}{9}\left(1-f_{\mathrm{s}}\right)^{2}+f_{\mathrm{s}}^{2} & \text { for } 0.4 \leq f_{\mathrm{s}} \leq 1 .\end{cases}$

The extra source vector in the gas flow equations $S_{\text {rad }}$ is given by:

$\boldsymbol{S}_{\mathrm{rad}}=r^{2} \sin \theta\left(\begin{array}{c}0 \\ \frac{\kappa}{c} F_{\mathrm{R}, r} \\ \frac{\kappa}{c} F_{\mathrm{R}, \theta} \\ \frac{\kappa}{c} F_{\mathrm{R}, \phi} \\ -\kappa c(B-E)\end{array}\right)$.

The total source term for the gas is given by $\boldsymbol{S}+\boldsymbol{S}_{\mathrm{rad}}$.

\section{Numerical method}

We use the technique of operator splitting to evolve the hydrodynamic part and the radiative part separately every time step.

\subsection{Hydrodynamics}

We numerically solve the flow equations for the gas using a three-dimensional version of the RODEO method (Paardekooper \& Mellema 2006b). For isothermal simulations the necessary eigenvalues, eigenvectors and projection coefficients are simple generalizations of the two-dimensional analogs given in Paardekooper \& Mellema (2006b). For simulations including an energy equation, the method is equivalent to the non-relativistic version of the general relativistic method of Eulderink \& Mellema (1995).

In short, RODEO evolves the Euler equations using an approximate Riemann solver (the Roe solver, see Roe 1981), together with stationary extrapolation to account for the geometrical and hydrodynamical source terms (Eq. (26)). The Coriolis forces are treated in an exact fashion to enforce exact angular momentum conservation (Kley 1998; Paardekooper \& Mellema $2006 \mathrm{~b})$. The extra source terms due to interaction with the radiation field (Eq. (38)) are treated as external source terms and are integrated separately (see below).

A module for adaptive mesh refinement (AMR) is used to obtain high resolution close to the planet. Because the planet remains at a fixed location on the grid, this treatment amounts to having a nested grid.

Accretion onto the planet is handled in the same way as in D'Angelo et al. (2003b): every time step $\Delta t$ the density near the planet is reduced by a factor $1-f \Delta t$. The area from which we take away mass has a size $r_{\text {acc }}$ times the Hill radius of the planet. For all accretion results in this paper, we have used $f=5 / 3$ and $r_{\text {acc }}=0.1$ (see D'Angelo et al. 2002). Klahr \& Kley (2006) used, in addition to runs with this accretion prescription, a procedure that conserves total energy locally by adding the kinetic and potential energy that is accreted onto the planet to the internal energy of the gas. Because we solve for the total energy of the gas directly, our accretion prescription does conserve total energy. This means that in the accretion region the gas is heated by the accretion process.

When calculating the torque on the planet, we exclude material that resides within the Hill sphere of the planet. This was 
also done in Bate et al. (2003). Although material inside the Hill sphere may exert a torque on the planet, which may lead to migration reversal (D'Angelo et al. 2003b), this torque is very sensitive to numerical resolution and the details of the accretion process. Moreover, this is also the region where self-gravity should play an important role, which is not included in our models. Therefore we focus on torques due to material outside the Hill sphere of the planet. We anticipate that for the low-mass planets that we consider in this paper this will not affect our results.

\subsection{Flux-limited diffusion}

Because of the intrinsic short time scale for radiative effects, which is due to the enormously fast communication speed $c$ compared to the sound speed, it is necessary to integrate the equation for $E$ in an implicit way.

First of all, consider the gas coupling term. We follow the approach described in Hayes \& Norman (2003). The gas is heated by the radiation field according to

$\frac{\partial \epsilon}{\partial t}=\kappa c(E-B)$,

where $\epsilon=p /(\Gamma-1)$ is the internal energy of the gas. Upon differencing both sides we obtain:

$\epsilon^{n+1}-\epsilon^{n}=\Delta t \kappa c\left(E^{n+1}-B^{n+1}\right)$,

where $X^{n}$ denotes quantity $X$ evaluated at time n. We approximate $B^{n+1}$ using a Taylor series expansion:

$B^{n+1} \approx a\left(T^{n}\right)^{3}\left(4 T^{n+1}-3 T^{n}\right)$,

and we spell out the internal energy as

$\epsilon^{n+1}=\frac{p^{n+1}}{\Gamma-1}=\frac{\rho \mathcal{R} T^{n+1}}{\Gamma-1}$,

in which $\mathcal{R}$ is the universal gas constant and $\Gamma$ is the adiabatic exponent. $T^{n+1}$ :

Putting Eqs. (42) and (41) into Eq. (40) we can solve for

$T^{n+1}=\frac{\epsilon^{n}+\Delta t \kappa c E^{n+1}+3 \Delta t \kappa c B^{n}}{\epsilon^{n}+4 \Delta t \kappa c B^{n}} T^{n}$.

This expression is used in Eq. (41) to evaluate $B^{n+1}$. We are now in a position to difference Eq. (34) (for details see Hayes $\&$ Norman 2003), which gives us an algebraic equation for $E_{i, j, k}^{n+1}$ at grid position $(i, j, k)$ :

$$
\begin{aligned}
& d_{i, j, k} E_{i, j, k}^{n+1}+d_{i+1, j, k} E_{i+1, j, k}^{n+1}+d_{i, j+1, k} E_{i, j+1, k}^{n+1} \\
& \quad+d_{i, j, k+1} E_{i, j, k+1}^{n+1}+d_{i-1, j, k} E_{i-1, j, k}^{n+1}+d_{i, j-1, k} E_{i, j-1, k}^{n+1} \\
& \quad+d_{i, j, k-1} E_{i, j, k-1}^{n+1}=Q_{i, j, k}^{n},
\end{aligned}
$$

for certain $d_{i, j, k}$. Note that we use only a seven point finite difference stencil. This is cheaper than taking into account all 26 neighboring cells, while we found no noticeable difference in the result.

We can write Eq. (44) as a linear system equation:

$\mathcal{D} \boldsymbol{E}^{n+1}=Q^{n}$,

in which $\mathcal{D}$ is a $N \times N$ matrix and $\boldsymbol{E}^{n+1}$ and $\boldsymbol{Q}^{n}$ are vectors with $N$ elements. Here, $N$ is the total number of grid points.
Fortunately, this huge matrix $\mathcal{D}$ is sparse; it has only seven nonzero entries every row. For such matrices efficient iterative linear solvers have been developed in the literature. For an overview see Barrett et al. (1994).

The most basic characteristic of a sparse linear solver is whether it is a stationary method or not. Stationary methods for solving Eq. (45) can be cast in the form:

$\boldsymbol{E}_{m}^{n+1}=\mathcal{A} \boldsymbol{E}_{m-1}^{n+1}+\boldsymbol{C}$

where $\boldsymbol{E}_{m}^{n+1}$ is the approximation to $\boldsymbol{E}^{n+1}$ after $m$ iterations. The matrix $\mathcal{A}$ nor the vector $\boldsymbol{C}$ depends on $m$ for a stationary method. Examples are the Jacobi method, the Gauss-Seidel method and successive over-relaxation (SOR), which was used for example in Klahr \& Kley (2006). The main disadvantage in using SOR lies in the fact that the convergence behavior of the method depends on a relaxation parameter $\omega$, for which the optimal value is problem-dependent.

Non-stationary methods are a more recent development, and they differ from stationary methods in that the structure of the update step changes with each iteration. A simple case is given by Eq. (46) with a matrix $\mathcal{A}$ and vector $\boldsymbol{C}$ that do depend on $m$. Examples of non-stationary methods are the conjugate gradient method (CG), the generalized minimal residual method (GMRES), the quasi minimal residual method (QMR) and the bi-conjugate gradient stabilized method (Bi-CGStab).

The optimal method for a given problem depends on the structure of the matrix $\mathcal{D}$. CG, for example, is only applicable to symmetric positive definite matrices, while we know in advance that due to opacity gradients the matrix $\mathcal{D}$ will not be symmetric. One could try CG on the matrix $\mathcal{D}^{\mathrm{T}} \mathcal{D}$, where $\mathcal{D}^{\mathrm{T}}$ is the transpose of $\mathcal{D}$, but this is not a good idea because the condition number of $\mathcal{D}^{\mathrm{T}} \mathcal{D}$ is the square of the condition number of $\mathcal{D}$, which would leads to very slow convergence of the method.

Another difference amongst the various methods is the memory requirement. When dealing with large matrices this can be a real bottleneck. For GMRES it can be shown that the optimal solution is reached within a finite number of iterations, but these iterations become more and more expensive because the method needs information from all previous steps. To avoid the enormous storage requirements one could decide to restart GMRES after a fixed number of iterations, but this introduces a free parameter in the method that needs to be tuned to the problem. Finally, QMR is more expensive in memory than Bi-CGStab in terms of memory because it needs more auxiliary variables. Therefore we have chosen Bi-CGStab as our method.

One potential disadvantage of Bi-CGStab is that it needs four inner products every iteration. An inner product is a global quantity, and therefore it requires communication between different processors, in addition to the boundary conditions. They act as synchronization points, and therefore load-balancing is critical in this part of the numerical method. We have implemented a dynamical load-balancing module, in which during execution of the code the processors exchange part of the computational domain to optimize the domain decomposition. Note that due to the AMR it is not true that every processor should get an equal part of the computational domain, because at an AMR boundary more work is needed (i.e. interpolation of data, flux correction).

We have preformed a strong scaling test on a low-resolution disk with a low-mass planet. The main grid consisted of 128 radial cells, 384 azimuthal cells and 8 meridional cells, on top of which we put 2 levels of AMR. The simulation was run on different numbers of processors $\left(n_{\text {proc }}\right)$ for a total of 5 orbits of the planet, which corresponds to approximately 7500 time steps. 


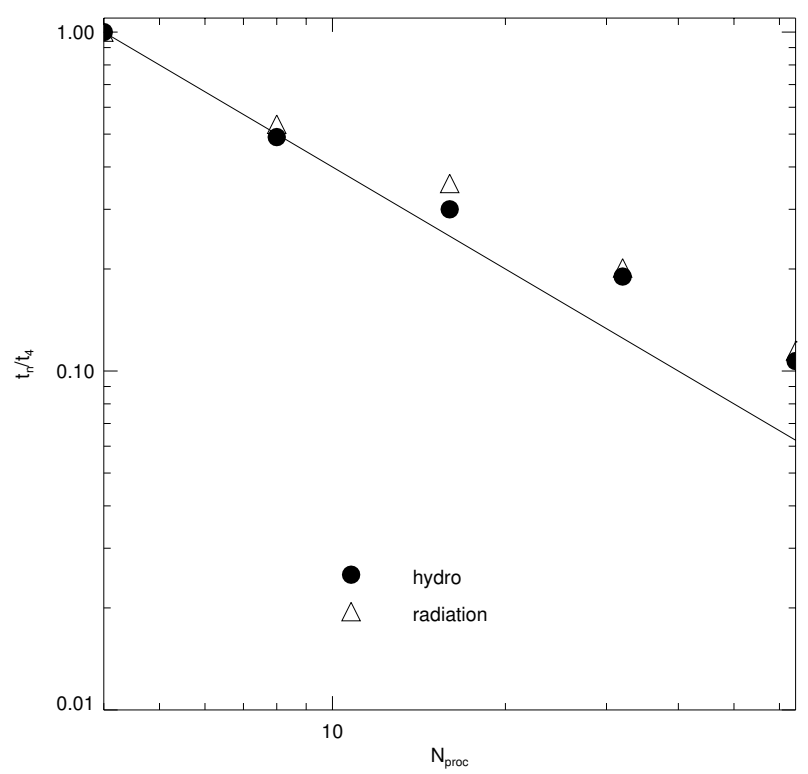

Fig. 1. Strong scaling test for the three-dimensional radiationhydrodynamical code, shown as the total execution time (relative to the execution time using four processors) as a function of the total number of processors. The solid line indicates perfect scaling, i.e. running on twice as many processors results in half the original execution time. Filled circles indicate the hydrodynamics part of the code; open triangles the radiation part. Note that both parts of the code scale equally well up to 64 processors.

The resulting total execution times, relative to the case of 4 processors, is shown in Fig. 1. Focusing on the hydrodynamical part first (filled circles), we see that the code scales perfectly up to $n_{\text {proc }}=8$. Due to memory requirements the simulation could not run on less than 4 processors, but the perfect scaling from 4 to 8 processors indicates that the code should scale very well from 1 to 4 processors as well.

When we increase $n_{\text {proc }}$ beyond 8 , the scaling is still very good, although not perfect. This is always the case at some point in a strong scaling test, because the amount of data that needs calculation stays the same, while the amount of data that has to be communicated increases with the number of processors. At some point increasing $n_{\text {proc }}$ does not result in a reduction in execution time anymore. Fortunately, for up to 64 processors we still measure a significant speed-up. This gives us confidence that for the high-resolution runs (that have 8 times more computational cells in the main grid) the scaling will be good up to $8 \times 64=512$ processors. The simulations presented in this paper were run on 126 processors of an SGI Altix 3700 CC-NUMA system with Intel Itanium-2 processors of $1.3 \mathrm{GHz}$. No use was made of the shared memory capacities of this system.

An interesting point in Fig. 1 is that the radiation module scales equally well as the hydrodynamics, even though the numerical methods for both parts of the physical problem differ significantly. This is an indication that for this problem only a small number of iterations is needed in the linear solver to reach convergence.

\subsection{Boundary conditions}

The planet excites waves in the disk, which subsequently propagate to the boundaries of the computational domain. To minimize reflection of outgoing waves we employ the non-reflective boundary conditions as outlined in Paardekooper \& Mellema (2006b) for the hydrodynamic variables. At the midplane we use reflective boundary conditions. No reflected waves were observed near the radial and upper meridional $(\theta=\pi / 2-$ $5 h / 2$ ) boundaries.

For the boundary conditions for the radiation energy density we take a different approach, because the initial condition for the gas temperature does not correspond to a stationary solution for $E$. In fact, due to the different opacity regimes at different heights above the midplane of the disk it is difficult, if not impossible, to obtain a stationary solution for $E$. In the case where the disk is optically thin throughout, the radiation energy density should vary as $E \propto r^{-2}$, which means that matter in equilibrium with this radiation has a temperature that varies as $T \propto r^{-1 / 2}$. In vertical hydrostatic equilibrium the relative scale height $h$ is then proportional to $r^{1 / 4}$, while the initial condition for the gas is a constant $h$. However, all disk models quickly (within a few orbits of the planet) adapt to a new equilibrium corresponding to the temperature structure set by the radiation field.

Real protoplanetary disks are heated internally by viscous dissipation (in regions where magnetic turbulence operates) and externally by radiation from the central star. Irradiation effects may play a large role in structuring protoplanetary disks (Dullemond 2000; Dullemond et al. 2001), but a detailed treatment of these effects is beyond the scope of this paper. However, in absence of heating sources the disk cools down rapidly (see Klahr \& Kley 2006), which will change the torque on embedded planets.

To account for irradiation in an approximate way we set the radiation energy density at the inner boundary to a constant value, which, for an optically this disk, would correspond to $h=0.05$ at the location of the planet. This way, we can compare the radiation-hydrodynamical simulations directly to their isothermal counterparts.

At the midplane we use reflective boundary conditions, while at the outer radial boundary we set the ghost cells such that $F_{\mathrm{R}, r} \propto r^{2}$, which corresponds to an outflow boundary. At the $\theta=\pi / 2-5 h / 2$ boundary we apply a similar procedure. This way, radiation can propagate freely off the grid.

\subsection{Test problems}

The RODEO hydrodynamics module was tested extensively on a Cartesian grid with shock tube problems: in all coordinate directions separately as well as in two dimensions diagonally. Also a genuinely two-dimensional problem, the wind tunnel with step, was studied and the results showed that the internal Roe solver is able to deal with the complex shock structures that arise in this problem (see also Mellema et al. 1991). On the two-dimensional disk-planet problem, RODEO was compared to other methods in de Val-Borro et al. (2006).

The radiation module was tested with one- and twodimensional diffusion problems with a simplified opacity law ( $\kappa=$ const.). See Stone et al. (1992) for a description of the diffusion tests. The numerical results were indistinguishable from the analytical solution.

Another test for the radiation solver is an optically thin circumstellar disk. Although FLD is in principle not very well suited for optically thin problems, it should nevertheless let $E$ diffuse towards the correct solution $E \propto r^{-2}$. Starting from the initial condition $E \propto r^{-4}$ (for $h=$ const., $T \propto r^{-1}$, therefore $E=a T^{4} \propto r^{-4}$ ) the numerical solution agrees with $E \propto r^{-2}$ within one orbit of the planet after which it remains stable. This is an important test, because it shows that we can compare the 
Table 1. Overview of model parameters. From left to right, the columns indicate: planet mass in $M_{\oplus}$, value of the adiabatic exponent $(\Gamma=1$ means a locally isothermal equation of state), viscosity parameter $\alpha$, radiation flag, accretion flag and the number of refinement levels. When $n_{\mathrm{lev}}$ levels of refinement are used, the local resolution within a distance of $2 H$ from the planet is a factor $2^{n_{\text {lev }}}$ higher than the base resolution of $\Delta r=0.0082 r_{\mathrm{p}}$. For the local models, the indicated resolution is valid for the whole computational domain.

\begin{tabular}{cccccccc}
\hline \hline$M_{\mathrm{p}}\left(M_{\oplus}\right)$ & $\Gamma$ & $\alpha$ & FLD & Acc & $n_{\text {lev }}$ & $\mathcal{S}$ & Comments \\
\hline 0.6 & 1 & 0.004 & $\mathrm{n}$ & $\mathrm{y}$ & 5 & 0 & \\
5 & 1 & 0.004 & $\mathrm{n}$ & $\mathrm{y}$ & 4 & 0 & \\
5 & 1 & 0.004 & $\mathrm{n}$ & $\mathrm{y}$ & 3 & 0 & \\
5 & 1 & 0.004 & $\mathrm{n}$ & $\mathrm{y}$ & 2 & 0 & \\
40 & 1 & 0.004 & $\mathrm{n}$ & $\mathrm{y}$ & 3 & 0 & \\
320 & 1 & 0.004 & $\mathrm{n}$ & $\mathrm{y}$ & 2 & 0 & \\
\hline 5 & 1.005 & 0 & $\mathrm{n}$ & $\mathrm{y}$ & 4 & -0.9925 & \\
5 & 1.01 & 0 & $\mathrm{n}$ & $\mathrm{y}$ & 4 & -0.9850 & \\
5 & 1.005 & 0 & $\mathrm{n}$ & $\mathrm{y}$ & 3 & -0.9925 & \\
5 & 1.01 & 0 & $\mathrm{n}$ & $\mathrm{y}$ & 3 & -0.9850 & \\
0.6 & 1.4 & 0 & $\mathrm{y}$ & $\mathrm{n}$ & 5 & -0.4 & \\
0.6 & 1.4 & 0 & $\mathrm{y}$ & $\mathrm{y}$ & 5 & -0.4 & \\
0.6 & 1.4 & 0 & $\mathrm{y}$ & $\mathrm{n}$ & 4 & -0.4 & \\
0.6 & 1.4 & 0 & $\mathrm{y}$ & $\mathrm{y}$ & 4 & -0.4 & \\
5 & 1.4 & 0 & $\mathrm{y}$ & $\mathrm{n}$ & 4 & -0.4 & \\
5 & 1.4 & 0 & $\mathrm{y}$ & $\mathrm{y}$ & 4 & -0.4 & \\
5 & 1.4 & 0 & $\mathrm{y}$ & $\mathrm{n}$ & 3 & -0.4 & \\
5 & 1.4 & 0 & $\mathrm{y}$ & $\mathrm{y}$ & 3 & -0.4 & \\
5 & 1.4 & 0 & $\mathrm{y}$ & $\mathrm{n}$ & 2 & -0.4 & \\
5 & 1.4 & 0 & $\mathrm{y}$ & $\mathrm{y}$ & 2 & -0.4 & \\
5 & 1.4 & 0 & $\mathrm{y}$ & $\mathrm{n}$ & 4 & -0.4 & 10 times lower density \\
5 & 1.4 & 0 & $\mathrm{y}$ & $\mathrm{n}$ & 4 & -0.4 & 100 times lower density \\
\hline 5 & 1.4 & 0 & $\mathrm{n}$ & $\mathrm{n}$ & 4 & -0.4 & local model \\
5 & 1 & 0 & $\mathrm{n}$ & $\mathrm{n}$ & 4 & 0 & local model \\
5 & 1.4 & 0 & $\mathrm{n}$ & $\mathrm{n}$ & 4 & -0.2 & local model \\
5 & 1.4 & 0 & $\mathrm{n}$ & $\mathrm{n}$ & 4 & 0.2 & local model \\
5 & 1.4 & 0 & $\mathrm{n}$ & $\mathrm{n}$ & 4 & 0.4 & local model \\
\hline & & & & & & & \\
& & & & & & & \\
\hline
\end{tabular}

radiation-hydrodynamical results in the optically thin limit directly to the isothermal runs.

Finally, we considered an optically thick disk with a constant opacity. In this case, the equilibrium solution is given by $E \propto$ $r^{-1}$. Again, starting from the initial condition for constant $h$ the numerical solution agrees with $E \propto r^{-1}$ within a few orbits of the planet, depending on the exact value of the opacity.

\section{Results}

In the previous sections we have described the physical and numerical setup for the three-dimensional radiationhydrodynamical simulations. However, we now move from the simple, 2D, viscous simulations toward the full models in two steps. The first step consists of 3D, isothermal simulations that are compared to Bate et al. (2003) and D'Angelo et al. (2003b). After that, we will include the energy equation but treat the cooling in a very simplistic way by setting the adiabatic exponent $\Gamma$ close to unity. Finally, we will present the full radiationhydrodynamical results. In Table 1 we give an overview of the simulations discussed in this paper.

\subsection{Isothermal models}

The flow structure around planets in three-dimensional isothermal disks has been described before (Bate et al. 2003; D'Angelo et al. 2003b). Therefore we limit the discussion to a few interesting remarks on the 3D-structure of the accretion flow. The disk model we use differs from the one described in Sect. 5 in two ways: we use a locally isothermal equation of state, and a kinematic $\alpha$-type viscosity (Shakura \& Sunyaev 1973) with $\alpha=0.004$ at the location of the planet. Essentially this model is the same as in D'Angelo et al. (2003b).

In Fig. 2 we show density slices along the three coordinate axis for a $5 M_{\oplus}$ planet after 10 orbital periods. By this time the flow has reached a steady state (Bate et al. 2003). For a lowmass planet like this the spiral wave features in the density are not strong, but they appear clearly in the velocity field.

From the middle and bottom panels of Fig. 2 we see that the material accreting onto the planet originates predominantly above and below the planet. As the material enters the Hill sphere of the planet, which for this mass is $0.017 r_{\mathrm{p}}$ large, it is accelerated to supersonic velocities. This was also observed by D'Angelo et al. (2003b), albeit not for a planetary mass as low as $5 M_{\oplus}$. Interesting is the equatorial outflow of mass that is apparent in Fig. 2. As the accreting material rains down on the planet it squeezes the envelope which forces some material to be expelled from the envelope in the equatorial plane of the disk. Even before leaving the Hill sphere of the planet this material again reaches supersonic velocities. It is important to note that no vertical hydrostatic equilibrium is established near the planet. This was also observed by D'Angelo et al. (2003b), even for non-accreting planets. The appearance of outflows does not depend on the boundary at $\theta=\pi / 2$ : we found the same behavior for a disk extending from $\theta=\pi / 2-5 h / 2$ to $\theta=\pi / 2+5 h / 2$. Therefore, this outflow is not linked to the boundary conditions.

In Fig. 3 we show the three-dimensional structure of the supersonic accretion and subsequent equatorial outflow. The solid volume represents the region where the Mach number of the flow, relative to the planet, is larger than one. For large radial distances to the planet the flow is always supersonic due to the Keplerian shear; this leads to the two large pizza boxes on both 

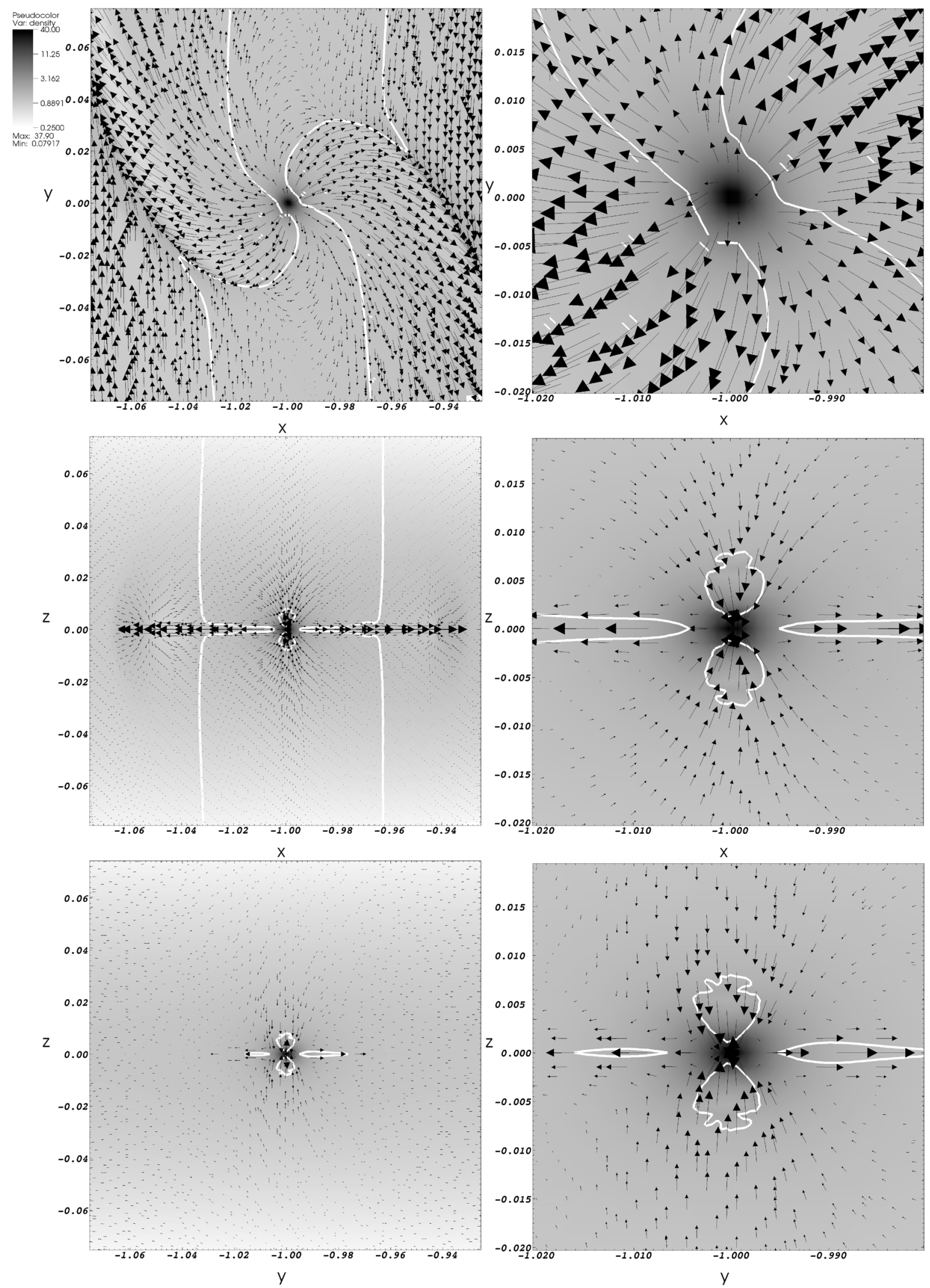

Fig. 2. Density and velocity fields around an accreting $5 M_{\oplus}$ planet after 10 orbits. Top panels: slice through $z=0(\theta=\pi / 2)$, middle panels: slice through $y=0(\phi=\pi)$, bottom panels: slice through $x=-1(r=1)$. Inside the white contour the velocities are supersonic.

radial sides of Fig. 3. The supersonic accretion velocities appear as the two small spheres just above and below the planet. The equatorial outflow is quickly turned into spiral wave features by the Keplerian shear. Note that the outflow is confined to the midplane of the disk. This is illustrated further in Fig. 4, where we show a density slice parallel to the midplane but at a height 


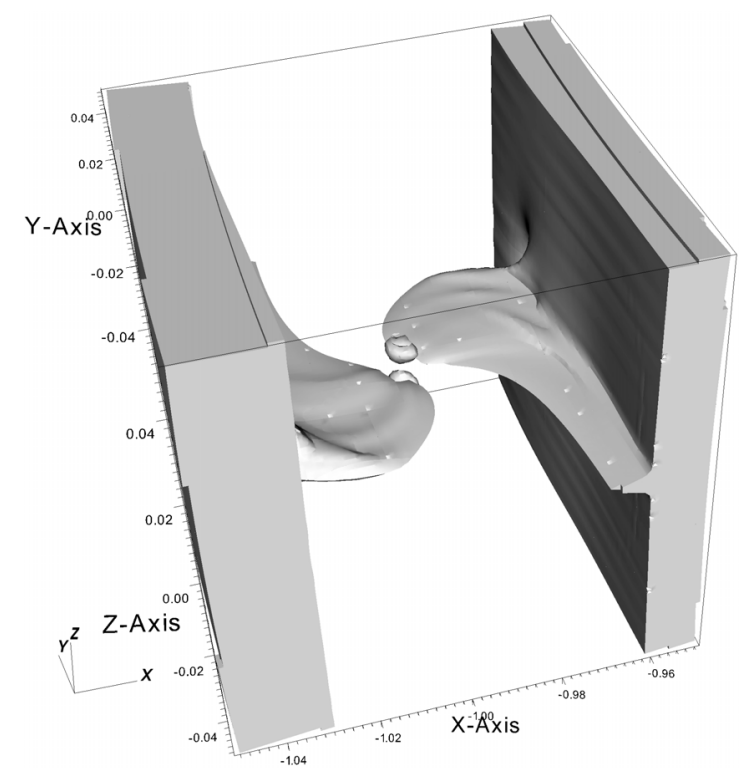

Fig. 3. Isovolume for a Mach number of 1. Inside the solid structures the velocity relative to the planet is supersonic.

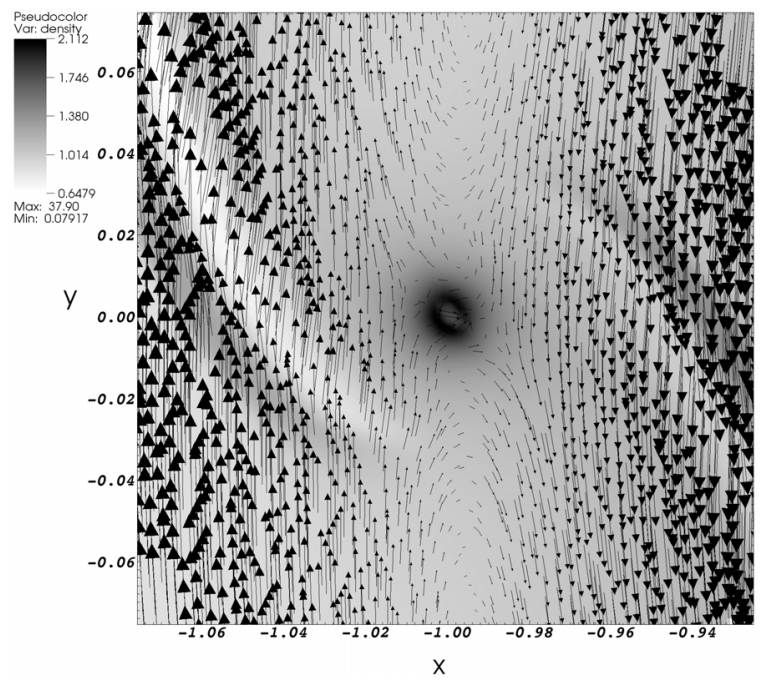

Fig. 4. Density and velocity fields around an accreting $5 M_{\oplus}$ planet after 10 orbits. The slice is taken at a distance of $0.005 r_{\mathrm{p}}$ from the mid plane of the disk.

of $0.005 r_{\mathrm{p}}$. Note that this is well within the Hill sphere of the planet. Even so, the velocity structure differs dramatically from that in the midplane. Apart from the unperturbed Keplerian velocity on both sides of the planet we can also see the horse-shoe orbits, as well as a rotating accretion flow close to the planet. This was also found by D'Angelo et al. (2003b).

The velocity structure inside the Hill sphere of the planet is intrinsically linked to the accretion procedure (D'Angelo et al. $2003 b)$. However, we found that this is not true for the equatorial outflow. It is there always when the vertical accretion flow is, albeit with a different magnitude for different accretion prescriptions.

For a planet of $0.6 M_{\oplus}$ the flow within the Hill sphere remains subsonic. The density and velocity field near the planet is shown in Fig. 5. Again we can see the vertical accretion flow from above and below the planet, some of which is deflected into the midplane of the disk where it may leave the Hill sphere of the planet. We can identify four circulation patterns near the edge of

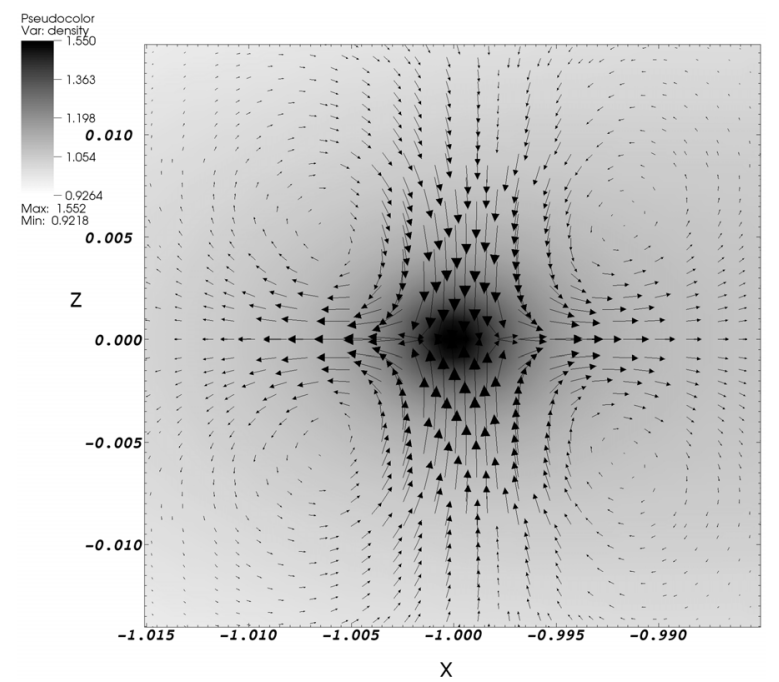

Fig. 5. Slice through $\phi=\pi$ for a $0.6 M_{\oplus}$ planet after 10 orbits, showing density and velocity. For this low-mass planet all velocities are subsonic.

the Hill sphere, which is approximately $0.009 r_{\mathrm{p}}$ large. Some of these patterns can also be seen in the results of D'Angelo et al. (2003b).

We will now turn to the question whether the differences in flow structure we find affect accretion and migration rates of embedded planets. In Fig. 6 we show the measured accretion rates as a function of planetary mass. Note that in this section we do not limit ourselves to low-mass planets in order to compare with previous numerical results (D'Angelo et al. 2003b). The twodimensional results, shown by the open symbols, were discussed extensively in Paardekooper \& Mellema (2006b) and we show them here just for comparison. We have modeled four different planetary masses, spanning a range from deep in the linear regime $\left(M_{\mathrm{p}}=0.6 M_{\oplus}\right)$ to well in the non-linear, gap-opening regime $\left(M_{\mathrm{p}}=1.0 M_{\mathrm{J}}\right)$.

Comparing the results of two- and three-dimensional simulations for the two planets with the highest mass, we find very good agreement. Especially for the $1 M_{\mathrm{J}}$ planets this is to be expected, because the Roche lobe of this planet is larger than the scale height of the disk, which makes the two-dimensional approximation applicable. But also for the planet of $0.1 M_{\mathrm{J}}$ we find good agreement, which contradicts the result of D'Angelo et al. (2003b). However, this discrepancy is small: for this planet, D'Angelo et al. (2003b) find a difference of approximately a factor of two between 2D and 3D results, while we find a difference of 1.3. It is possible that because our method is specifically designed to handle shocks in a correct way, non-linear effects start to play a role for slightly lower planetary masses. Our results on migration rates support this suggestion (see below).

Because the Roche lobes of the low-mass planets are much smaller than the disk thickness the two-dimensional approximation breaks down. This affects migration as well as accretion, but the effect on the accretion rates is the most dramatic. When in the two-dimensional approach mass is taken away from the location of the planet, what essentially happens is that a whole column of gas is accreted onto the planet. In the full threedimensional problem this does not happen, and therefore the accretion rates are significantly lower. For the smallest planet we consider $\left(M_{\mathrm{p}}=0.6 M_{\oplus}\right)$ the difference amounts to more than three orders of magnitude (see the right panel of Fig. 6). 


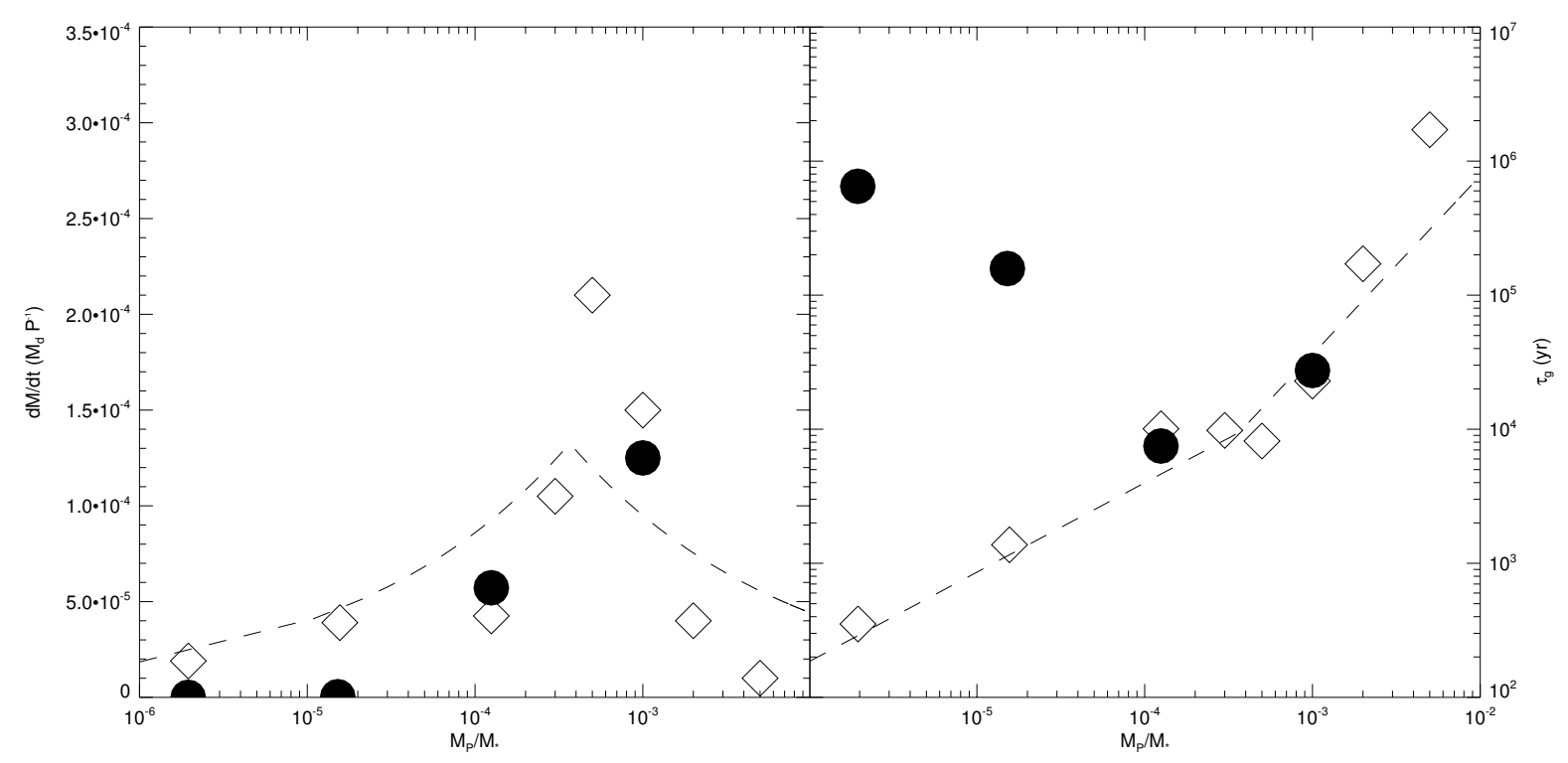

Fig. 6. Accretion rates for two- and three-dimensional isothermal simulations. Open symbols represent the two-dimensional results from Paardekooper \& Mellema (2006b), filled symbols denote the three-dimensional results. Left panel: accretion rate in units of disk masses per orbit for different planetary masses. Right panel: growth time scales $\tau_{\mathrm{g}}=M_{\mathrm{p}} / \dot{M}_{\mathrm{p}}$ in years, where we assume a disk mass of $5 M_{\mathrm{J}}$.

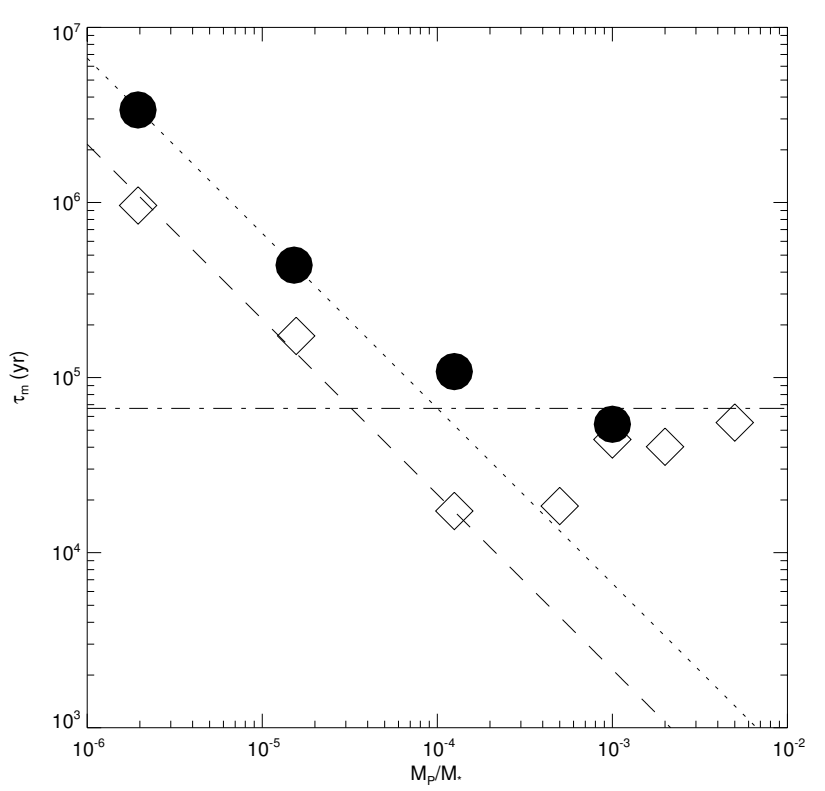

Fig. 7. Migration time scales $\tau_{\mathrm{m}}=r_{\mathrm{p}} / \dot{r}_{\mathrm{p}}$ for two- (open symbols) and three-dimensional (filled circles) isothermal simulations. As in Fig. 6 we assume a disk mass of $5 M_{\mathrm{J}}$. The dotted line and the dashed line indicate the analytical results from Tanaka et al. (2002) for type I migration in three-dimensional and two-dimensional disks, respectively. The horizontal dash-dotted line indicates migration on the viscous time scale of the disk (type II migration).

Qualitatively our results agree very well with the results of D'Angelo et al. (2003b). Interestingly, Bate et al. (2003) do not find an increase in growth time towards the low-mass end, but this is probably due to a lack of resolution. Our results show that in order to capture significant amounts of gas the mass of a solid core needs no be at least several Earth masses.

Migration rates also differ between 2D and 3D disks (Tanaka et al. 2002). Again, the difference is most pronounced for lowmass planets, while for planet with masses compared to Jupiter the two-dimensional approximation gives valid results. In Fig. 7 we show the migration time scales as a function of planetary mass. For a planet on a circular orbit, the migration time scale is related to the torque in the following way:

$$
\frac{1}{\tau_{\mathrm{m}}}=\frac{\left|\dot{r}_{\mathrm{p}}\right|}{r_{\mathrm{p}}}=\frac{2|\dot{L}|}{L}=\frac{2|\mathcal{T}|}{L},
$$

where $L$ is the angular momentum of the planet and $\mathcal{T}$ is the torque, which is what we measure during the simulations.

Again, for the $1 M_{\mathrm{J}}$ planet the 2D result agrees very well with the $3 \mathrm{D}$ result, for the same reason as the accretion rates. In fact, they approach the migration rate dictated by the viscous evolution of the disk (type II migration). On the low-mass end, we can nicely reproduce the analytical results from Tanaka et al. (2002) for type I migration in $2 \mathrm{D}$ as well as in $3 \mathrm{D}$. In this regime, $3 \mathrm{D}$ migration time scales are approximately a factor of two larger than for 2D disks.

In between the two regimes, we find that for a $0.1 M_{\mathrm{J}}$ planet $2 \mathrm{D}$ and $3 \mathrm{D}$ results differ significantly, almost an order of magnitude. This is again in contradiction with D'Angelo et al. (2003b), but note that the main difference lies in the $2 \mathrm{D}$ results. These differences were already discussed in Paardekooper \& Mellema (2006b) and we will not repeat that discussion here. We only note that the results for a three-dimensional disk agree very well with D'Angelo et al. (2003b). Also, the departure from the type I analytical result for the $0.1 M_{\mathrm{J}}$ planet indicates that for this planet non-linear effects start to play a role, in accordance with the suggestion made above that these effects are also visible in the accretion rates.

Summarizing, we find excellent agreement with analytical results of torques in the low-mass regime as well as in the highmass regime. In between, the results are not clear but D' Angelo et al. (2005) showed that this may be a general problem for this transition regime. The measured accretion rates agree very well qualitatively with D'Angelo et al. (2003b), again with the exception of the transition regime between linear and non-linear interaction with the disk. 


\subsection{Nearly-isothermal models}

As a bridge between the simple, locally isothermal models of the previous section and the full radiation-hydrodynamical simulations we now consider models that do include the energy equation, but in which we treat the cooling in a simplified way. Consider the relation between internal energy and pressure for a perfect gas:

$p=(\Gamma-1) \epsilon$,

which shows that the adiabatic exponent $\Gamma$ is a measure of how a change in internal energy affects the gas pressure, and, given the density, therefore also the temperature of the gas. For the isothermal case, $\Gamma=1$, pressure and internal energy evolve independently and the gas can be compressed indefinitely without changing the temperature. To simulate cooling in a very simplified way one can take a value of $\Gamma$ very close to 1 , which was done by Nelson \& Benz (2003a,b) for the two-dimensional planet-disk problem. In Paardekooper \& Mellema (2006b) we commented already that this approach affects planetary accretion rates to a large extent, because even for $\Gamma=1.001$ the planet forms a hot bubble around itself that prevents matter from entering the Roche lobe.

In Fig. 8 we show the density and velocity structure around a $5 M_{\oplus}$ planet after 10 orbits. This figure can be directly compared to the isothermal model depicted in Fig. 2. The global properties of the velocity field are similar to the isothermal case: inflow from above and below the planet and outflow in the midplane of the disk. Both inflow and outflow are again supersonic.

The central density is approximately 2 times lower in the nearly-isothermal model compared to the fully isothermal case. This is due to the fact that it is not only the density gradient that determines the pressure gradient, but also the temperature. This means that part of the pressure-support for the planetary envelope comes from the temperature gradient. We will see below that this lower central density substantially affects the accretion rate onto the planet.

Another important difference that can be seen in the top left panels of Figs. 2 and 8 is the change in outflow structure. While in the isothermal case of Fig. 2 both outflow streams appear to be symmetric this is no longer the case when changes in temperature are taken into account. In the top right panel of Fig. 8 we see that the outflow towards positive $y$ appears to be much more collimated along the $y$-axis than the outflow towards negative $y$, which is responsible for the asymmetry seen in the top left panel of Fig. 8. It is important to realize at this point that these asymmetries may suffer from resolution effects. Although in these simulations we resolve the Roche lobe by approximately 50 cells, the high-density region inside of the outflow is only resolved by 5 cells. Resolution studies show that the direction of the outflow does not depend on resolution, but the magnitude of the outflow does. This is because the central temperature and density also scale with resolution, as the minimum distance to the planet does, and therefore also the depth of the potential well. The equatorial outflow therefore critically depends on the conditions near the center of the planet where the density and the temperature reach their highest values. Therefore there exists a connection between the conditions deep in the atmosphere of the planet and the planet's immediate surroundings.

In Fig. 9 we show the temperature structure around the same planet as in Fig. 8. In this model, the central temperature has risen from $50 \mathrm{~K}$ initially to more than $100 \mathrm{~K}$. Note that it is only a very small region that acquires this higher temperature. This reflects the fact that the accreting region is only $10 \%$ of the planet's Roche lobe. In this region, the gas is heated by the release of potential and kinetic energy of the accreted gas. The two streams of outflowing material along the $y$-axis are clearly visible due to their high temperature. Also the collimation of the stream in the positive $y$-direction is apparent in the top left panel of Fig. 9. The outflows remain confined to the midplane of the disk until they reach the spiral waves induced by the planet that are responsible for upward motion of gas. However, for a lowmass planet this upward motion is not strong, and from the top right panel of Fig. 9 we see that the hot flow does not reach high altitudes.

Another important effect can be seen in the bottom right panel of Fig. 9 that shows the three-dimensional temperature distribution around the planet. The solid volume indicates temperatures higher than the initial temperature at the location of the planet. The large solid structure at $x>-1$ is due to the global temperature gradient. To the left of this structure we see the effects of the planet, especially the spiral shaped hot outflow. But note that the corotational region located at $x=-1$ is also asymmetrically heated: for positive values of $y$ the corotation region is locally heated by the planet, while this does not happen for negative $y$. Again, this is a consequence of the asymmetric hot outflow that originates deep inside the envelope of the planet. When the pressure remains the same, however, this temperature distribution leads to an asymmetric density distribution which will affect the total torque on the planet. Because the region behind the planet is heated compared with the region in front of the planet, the density will be relatively low behind the planet which leads to a torque that will be positive if the effect is strong enough. We will see below that for this model indeed the total torque on the planet is positive.

Before looking at the torques onto the planet we consider the measured accretion rates. In Fig. 10 we show the accretion rate as a function of time for three different equations of state: the isothermal result discussed in Sect. 7.1 together with two nearlyisothermal models. It turns out that the accretion rate depends sensitively on the central temperature. This is not surprising, because the pressure in near the center of the planet is determined mainly by the potential of the planet, which remains the same. Therefore, when the central temperature rises by a factor of 2 , the central density will be a factor of 2 lower which in its turn affects the accretion rate onto the planet. From Fig. 9 we saw that for $\gamma=1.01$ the central temperature indeed was a factor 2 higher than the initial temperature, while from Figs. 2 and 8 we see that the central density is lower by approximately a factor 2 for the model with $\Gamma=1.01$ compared to the isothermal model. In Fig. 10 we see that this change in density is reflected in an accretion rate that is lowered again by a factor 2 .

When we take $\Gamma$ closer and closer to 1 , we expect to retrieve the isothermal results. However, as was already mentioned in Paardekooper \& Mellema (2006b), even for $\Gamma$ extremely close to 1 the central temperature of the planet rises significantly. In fact, due to the necessary divisions by the factor $\Gamma-1$ (see Eulderink \& Mellema 1995) one has to worry about numerical stability for $\Gamma \approx 1$. Therefore the lowest value of $\Gamma$ we considered is $\Gamma=1.005$. For this model, the central temperature rises to approximately $90 \mathrm{~K}$, which is again reflected in a lower accretion rate than for the isothermal model (see Fig. 10).

In Fig. 11 we show the total torque onto the planet for the same models as in Fig. 10. The torques are normalized to the absolute value of the analytic result of Tanaka et al. (2002), and as was already shown in Fig. 7 for the isothermal model we find very good agreement with the analytical result. This can not be said of the nearly-isothermal models, however. Indeed, for both 

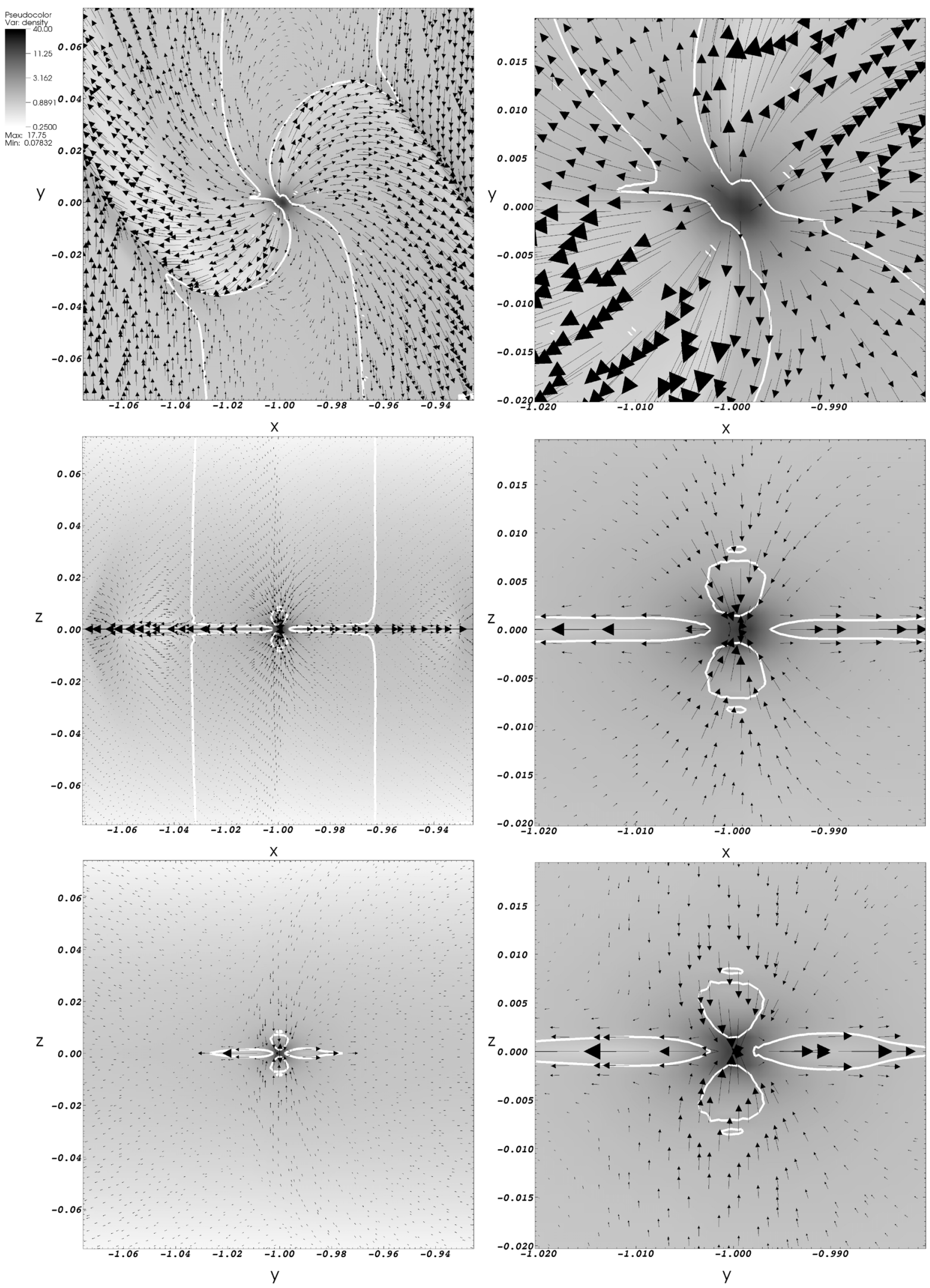

Fig. 8. Same as Fig. 2, but for a nearly isothermal model with $\Gamma=1.01$.

values of $\Gamma$ we considered we find a positive torque, indicating outward migration. But not only is the direction reversed, also the magnitude of the torque is surprising: more than six times larger than for the isothermal case. The actual value of $\Gamma$ has no influence on this result. Note that it takes a few orbits longer for the nearly-isothermal models to reach a steady state with respect to the torque, which was not the case for the accretion rates. This is probably due to the fact that the torque is modified by the 


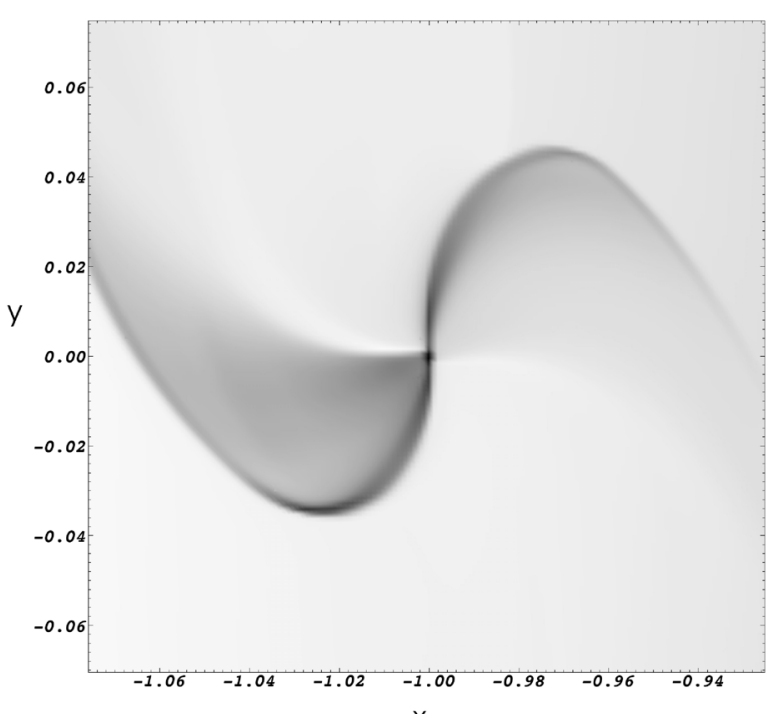

$\mathrm{X}$

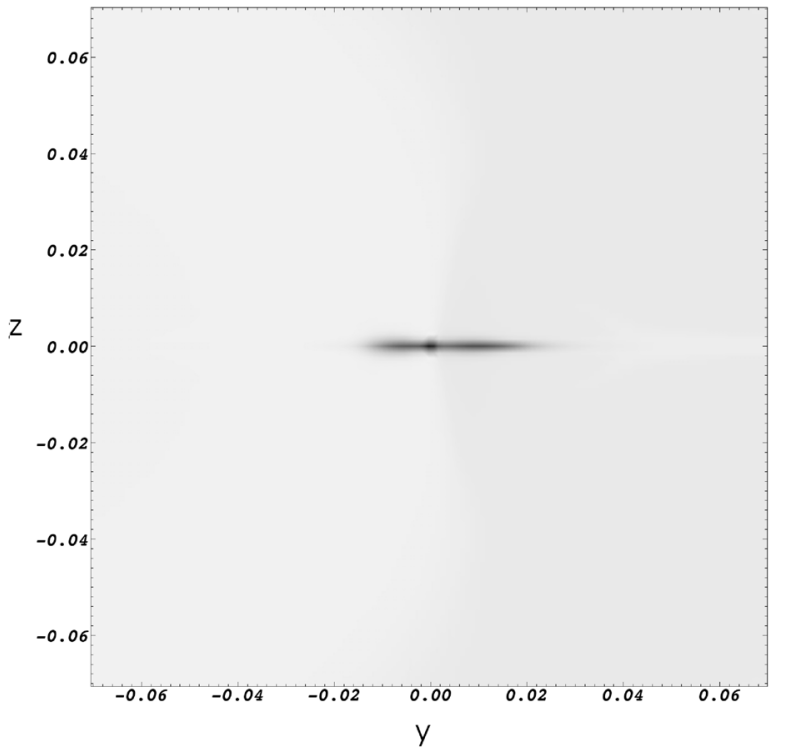

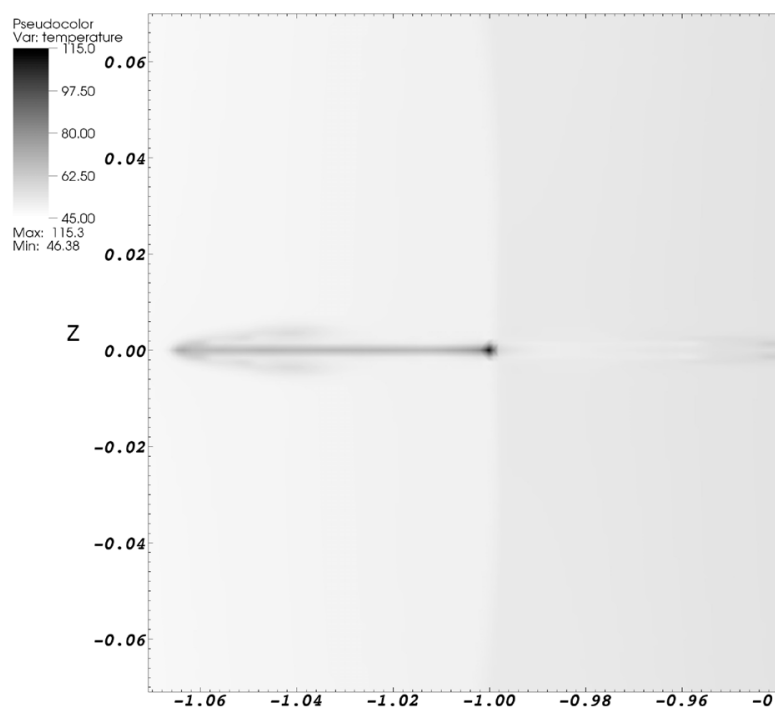

$\mathrm{X}$

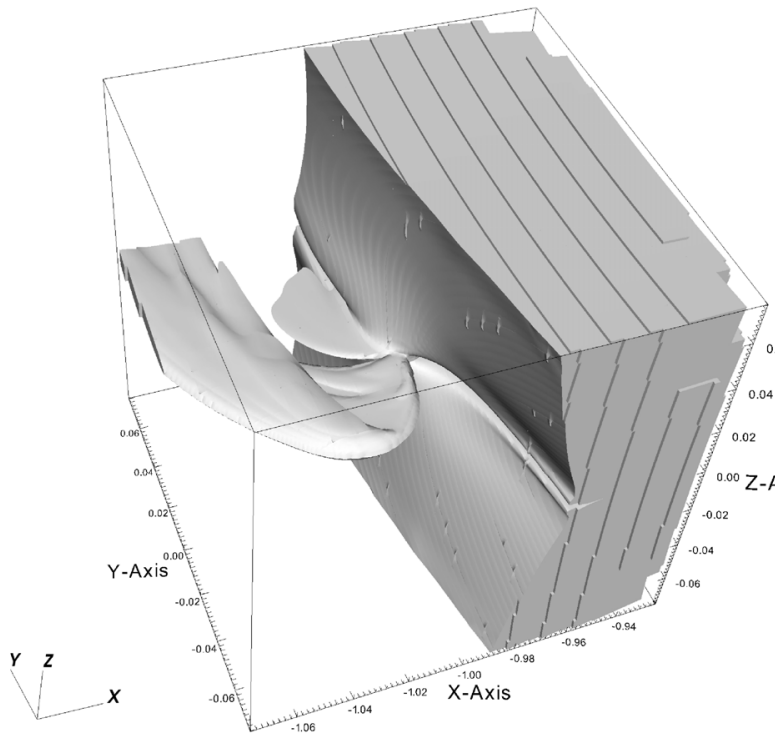

Fig. 9. Temperature structure around a $5 M_{\oplus}$ planet after 10 orbits for $\Gamma=1.01$. Top left panel: slice through $z=0$ (equatorial plane). Top right panel: slice through $y=0(\phi=\pi)$. Bottom left panel: slice through $x=-1(r=1)$. Bottom right panel: isovolume indicating a temperature higher than the initial temperature at the location of the planet.

equatorial outflow. This material has to come close to the planet which takes a few orbits.

It is interesting to find out where exactly this large positive contribution to the torque originates. In order to answer this question, we show in Fig. 12 the radial torque profile for the three different equations of state. For the isothermal case we see the usual (see Bate et al. 2003; D'Angelo et al. 2003b) contributions from the inner and the outer spiral wave, where the inner wave exerts a positive torque on the planet and the outer wave a negative torque. It is clear that even from inspection by eye that the torque due to the outer wave is slightly stronger which leads to inward migration.

The situation is totally different for nearly-isothermal models, as can be seen in Fig. 12. For the outer disk the changes are marginal, which is not surprising because the structure of the outer wave in Figs. 2 and 8 appears very similar. This in contrast with the inner wave, which has a much more open structure for the nearly isothermal model compared with the truly isothermal model. This changing wave structure leads to a net positive torque on the planet.
From the temperature plots in Fig. 9 we conclude that the outflowing material originates deep within the Roche lobe of the planet. This is an indication that accretion and migration rates of forming planets are closely linked. This was already suggested by D'Angelo et al. (2003b) for the isothermal case. However, only when the energy equation is taken into account the migration behavior changes this drastically. Although in this section we have treated cooling in a simplified way, it provides useful insight in what we may expect from the full radiationhydrodynamical models. In the next section, we will consider a more realistic treatment of the energy balance by explicitly including radiative cooling.

\subsection{Radiation-hydrodynamical models}

The full radiation-hydrodynamical models differ from the nearly-isothermal models in two ways: first of all, the adiabatic exponent is set to $\Gamma=1.4$, which reduces the compressibility of the gas. Second, the FLD module calculates the energy diffusion 


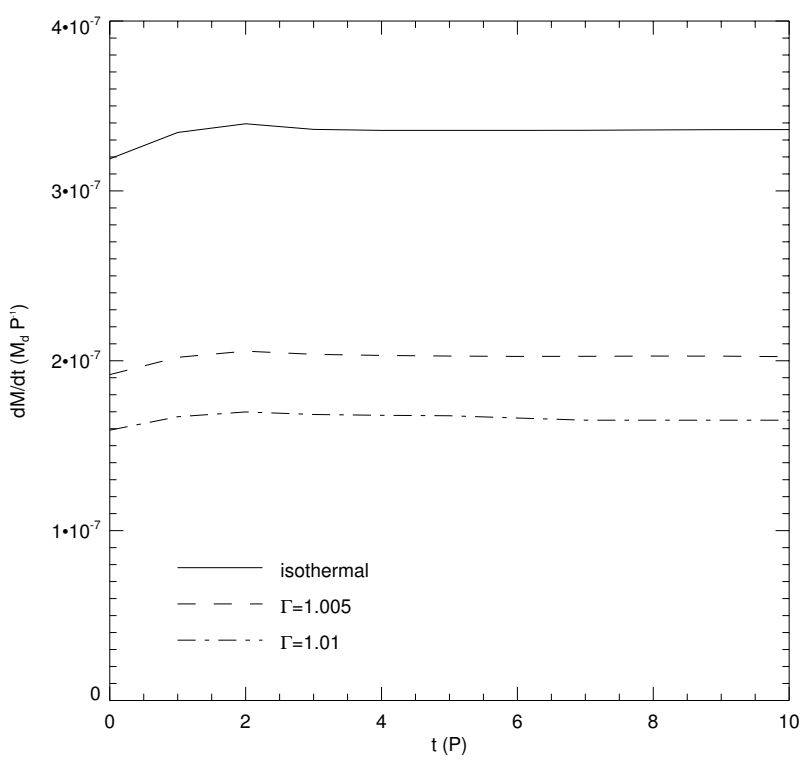

Fig. 10. Accretion rates onto a $5 M_{\oplus}$ planet for three different equations of state.

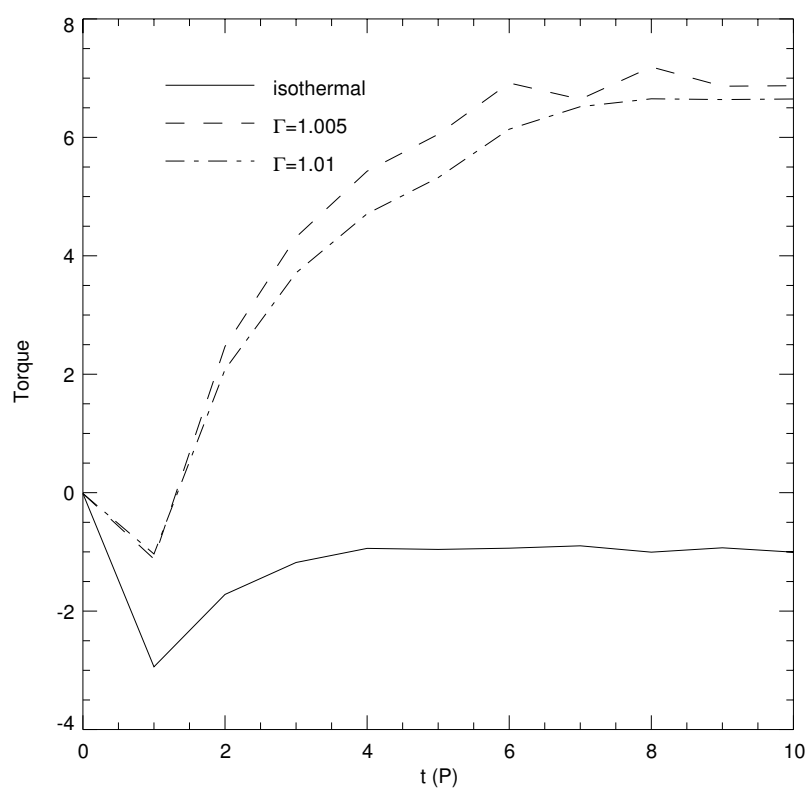

Fig. 11. Total torque onto a $5 M_{\oplus}$ planet for three different equations of state. The torques are normalized to the absolute value of the analytical result of Tanaka et al. (2002).

through radiation. Therefore, the cooling properties of the gas depend on the local temperature and density. When cooling is not efficient, we expect that due to the higher value of $\Gamma$ the effects seen in the nearly-isothermal models will be enhanced even more. This will certainly happen in the high density envelope of the planet, and because we saw in the previous section that temperature effects deep within the Roche lobe of the planet affect the migration behavior we may expect to see similar things in the radiation-hydrodynamical models.

In order to distinguish the effects from the accretion itself from the overall radiation-hydrodynamical behavior of the disk, we consider two cases in this section: an accreting $5 M_{\oplus}$ planet and a non-accreting planet of the same mass. The non-accreting planet is allowed to build a high-density atmosphere because no mass is removed from the Roche lobe. However, as was already

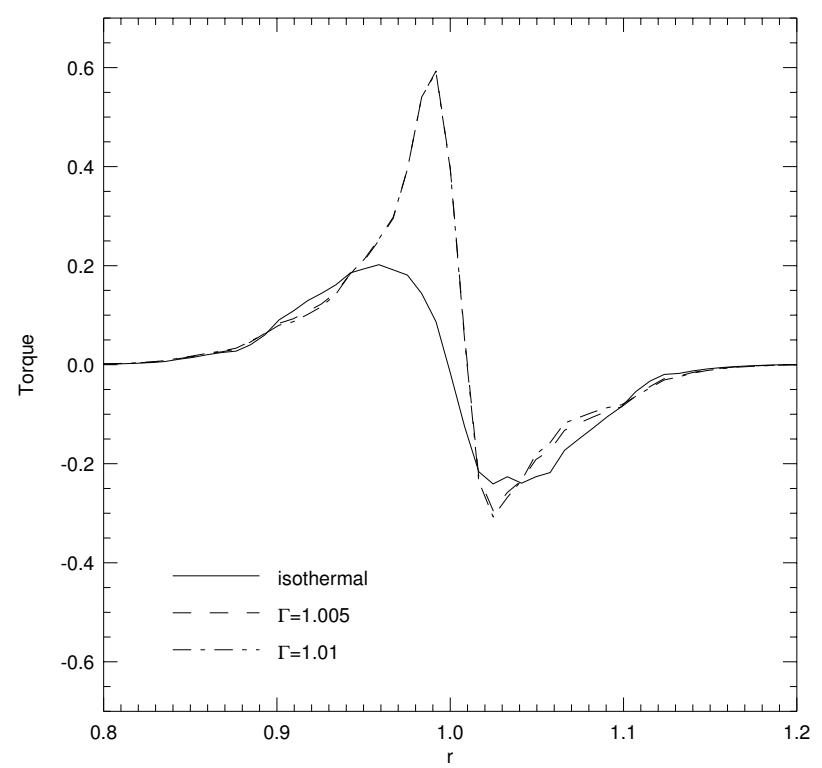

Fig. 12. Radial torque profile for a $5 M_{\oplus}$ planet for three different equations of state. The torques are normalized in such a way that the total torque for an isothermal equation of state is equal to -1 .

shown by D'Angelo et al. (2003b) for the isothermal case, even in this case the envelope does not reach a state of hydrostatic equilibrium in the vertical direction. Material still rains down into the Roche lobe from above and below the planet, some of which subsequently flows out again. Thus even in the case of a non-accreting planet there may exist a close connection between the state of the material deep within the planetary envelope and the torques due to material outside the Roche lobe.

In Fig. 13 we show the density and velocity structure for the non-accreting planet of $5 M_{\oplus}$. The color scale is the same as in Figs. 2 and 8, which tells us immediately that the density of the planetary envelope is very low compared to the isothermal and nearly-isothermal cases. This reflects the effect of the lower compressibility of the gas. We will see below that this has dramatic effects on the ability of the planet to accrete gas from the disk.

Looking at the velocity field in Fig. 13 we see that indeed there is no hydrostatic equilibrium in the vertical direction. The same flow pattern appears as in the isothermal and nearly-isothermal cases: inflow from above and below and outflow along the equator of the planet. Inflow velocities are lower in this case because the planet does not accrete matter (D'Angelo et al. 2003b). The outflow appears much more symmetric in all directions compared to the previous nearly-isothermal case. Therefore radiative cooling restores the isothermal situation in these regions of lower density than the planetary core. However, we will see that still important differences arise in the torque on the planet.

In Fig. 14 we show the temperature structure around the planet, on the same scale as in Fig. 9. The most important difference with the midplane temperature structure of the nearlyisothermal models is the absence of the accretion jets because in Fig. 14 we show a non-accreting model. The temperature at the center of the planet is also lower; about a factor of 2 higher than the initial temperature. This agrees with Eq. (17) for the maximum central temperature that can be reached in absence of convection. We can also identify the spiral waves in the temperature structure, because when the gas is compressed in the wave the temperature rises. In the dense midplane of the disk the gas can 

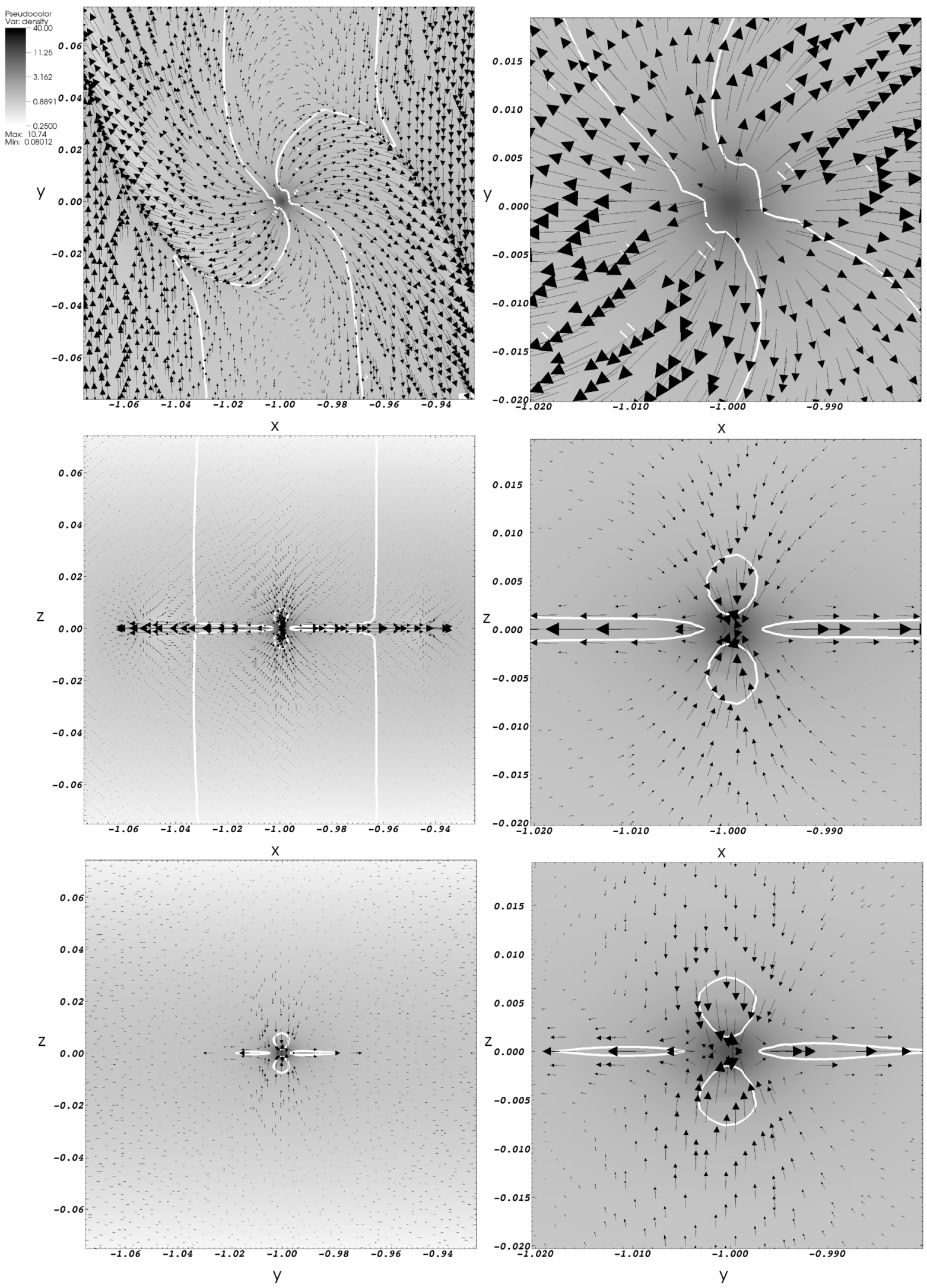

Fig. 13. Same as Fig. 2, but for a non-accreting radiation-hydrodynamical model with $\rho_{0}=10^{-11} \mathrm{~g} \mathrm{~cm}^{-3}$.

not radiate away this energy efficiently, and therefore the spiral waves have a higher temperature.

Inside the equatorial outflow the gas expands, and therefore cools down. This effect causes the temperature close to the hot core to be lower than the initial temperature. These regions show up as dark areas in Fig. 14, most notably in the top right panel.

Another difference with the nearly-isothermal models can be spotted most clearly in the bottom-left panel of Fig. 14. While 

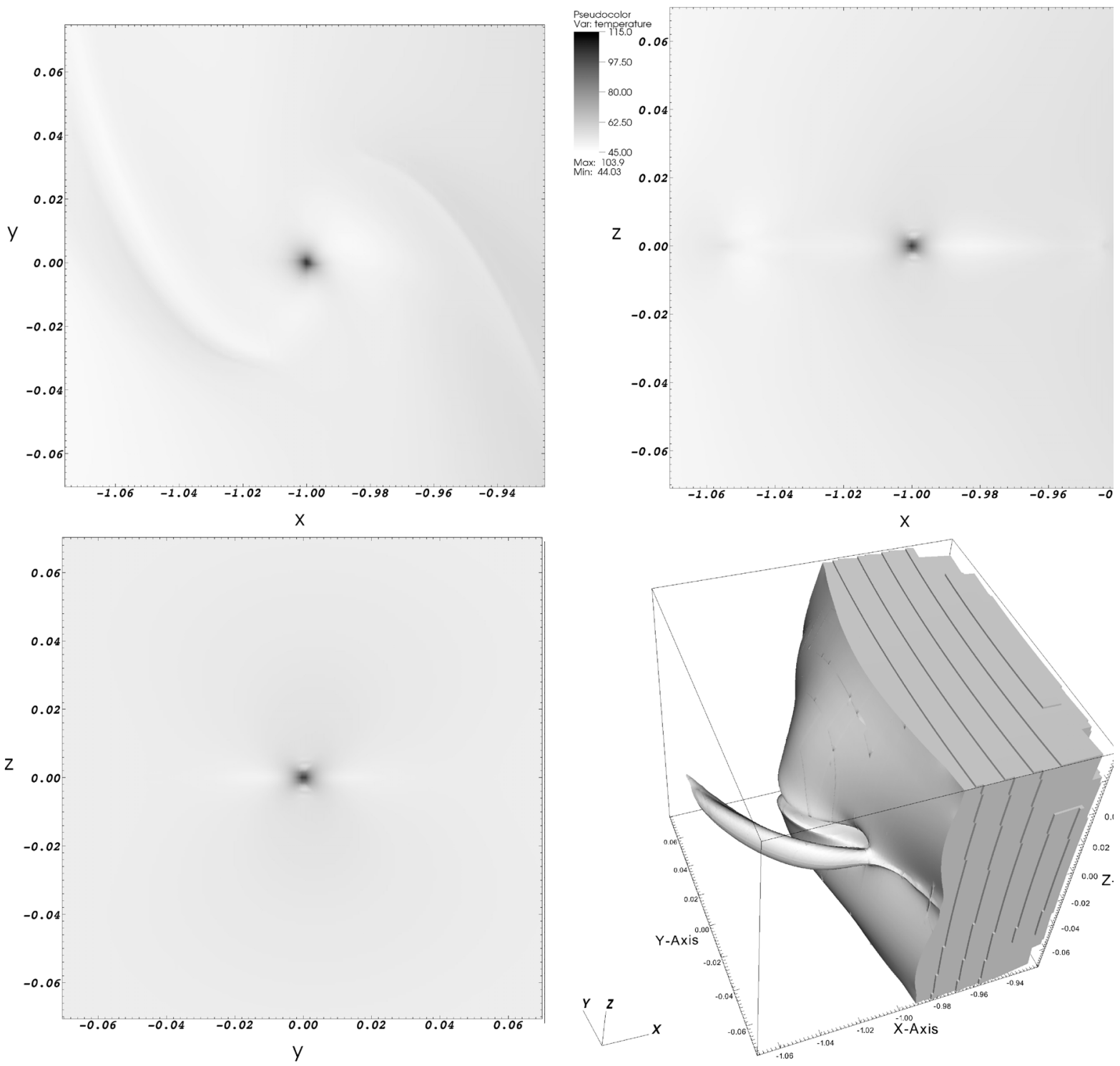

Fig. 14. Same as Fig. 9, but for a non-accreting radiation-hydrodynamical model.

the heat generated in the envelope of the planet remains confined to the midplane of the disk in the nearly-isothermal models (see Fig. 9), in the radiation-hydrodynamical models it can diffuse to higher altitudes. Because of the large opacity gradient in the vertical direction, this is the preferable direction for radiative diffusion.

In the bottom right panel of Fig. 14 we again, just as in Fig. 9, show a 3D isovolume of the temperature structure around the planet. It is clear that also the spiral wave structure in the temperature extends further in the vertical direction due to radiative heat diffusion. Also it is clear that the strong accretion jets are absent. However, what remains is the asymmetric temperature structure in the corotation region. There is a bump visible for positive $y\left(\phi<\phi_{\mathrm{p}}\right.$, the region behind the planet) while there is a small hole for negative $y$. This asymmetric corotational temperature structure affects the corotation torque.

In Fig. 15 we show the temperature and velocity structure for the case of an accreting planet. It is immediately clear that the temperature is raised considerably in the planetary envelope due to the accretion proces. Note that in this case the planetary envelope is unstable to convection according to Eq. (17): the heat generated in the accretion process can not be transported to the surface of the Hill sphere by radiation alone. Although Eq. (17) is of limited applicability because of the assumption that the local pressure scale height is the same as for the total disk, we can identify heat transport by fluid motion in the equatorial plane of the disk. The top left panel of Fig. 15 shows that the outflow velocity pattern is changed with respect to the non-accreting model: it seems that heat flows preferentially into the outer disk. However, it should be stressed that this observed asymmetry may be caused by insufficient resolution at the very center of the planetary envelope. Nevertheless, these hot outflows cause significant changes in the temperature and density distribution in the immediate surroundings of the planet and are therefore potentially important for the migration behavior of the planet. The details of the accretion process should therefore be subject of further study.

Also from Fig. 15 we see that the convective flow is confined to the midplane of the disk: vertical velocities in the top right and bottom left panel are all directed towards the planet. This 

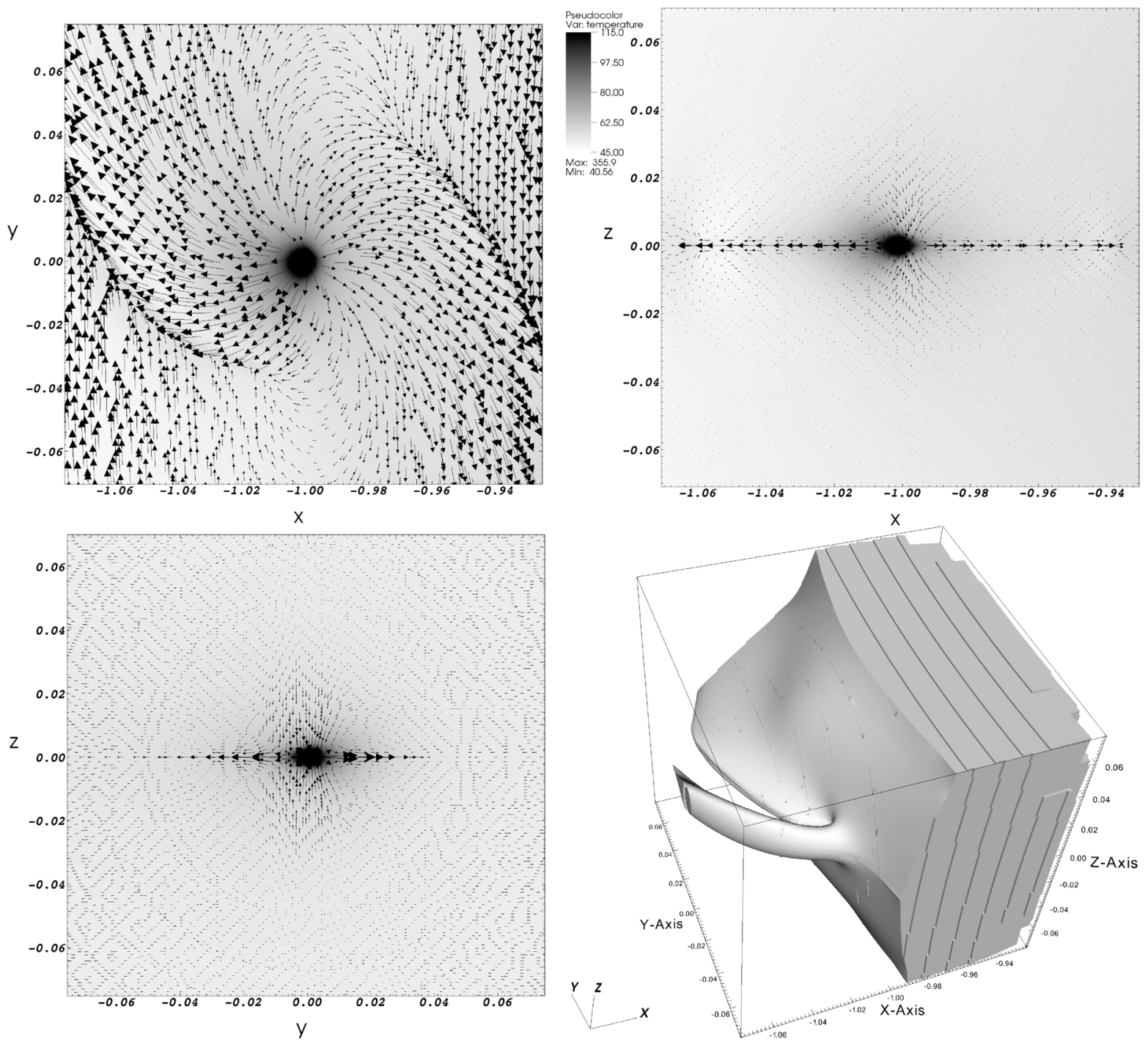

Fig. 15. Same as Fig. 9, but for an accreting radiation-hydrodynamical model. Black arrows indicate the velocity field.

shows that the vertical thickness of the hot bubble surrounding the planet is due to radiative heat diffusion. Its vertical extension is roughly the size of the Hill sphere of the planet.

The formation of the hot bubble has dramatic consequences for the measured accretion rate onto the planet. In Fig. 16 we show the accretion rate as a function of time for the isothermal case (dotted line) and two radiation-hydrodynamical models with two different initial values for the density at the location of the planet. The solid line indicates an initial density of $\rho_{0}=10^{-11} \mathrm{~g} \mathrm{~cm}^{-3}$ which is our nominal value. For this density we measure an accretion rate that is more than an order of magnitude lower than for the locally isothermal equation of state. The hot bubble surrounding the planet severely limits the gas flow towards the planet. Because this bubble is created by the accretion process itself, accretion is a self-limited process in this case.

For a lower density the cooling efficiency increases. In fact, according to Eq. (10) the cooling time is proportional to the square of the density. For a model with a ten times lower density (dashed line in Fig. 16) we measure an accretion rate that is almost ten times higher than for our nominal model. This accretion rate is comparable to the results for nearly-isothermal models (see Fig. 10). Lowering the density even more did not change the accretion rate significantly. This also agrees with the nearly-isothermal models, where we saw that even for $\Gamma$ as low as 1.005 the accretion rate remained far from the isothermal result. This can be understood in terms of the high central density required to achieve the isothermal accretion rate. From Fig. 2 we see that the central density is more than a factor 37 higher than the initial density. It is very hard to cool such a high-density envelope efficiently, especially when accretion-generated heat is considered as well.

In Fig. 17 we show the growth time scales for the isothermal results (see also Fig. 6) with the radiation-hydrodynamical results added as open triangles. The changes with respect to the isothermal models are not as dramatic as the changes from moving from 2D simulations to 3D simulations, but still it represents another order of magnitude slower accretion. Also from Fig. 17 we see that the effect is slightly less strong for a planet of $0.6 M_{\oplus}$. This is because the potential for this planet is less deep, but the decrease in accretion rate is still significant even for this very small planet.

We now turn to the question of planetary migration within the radiation hydrodynamical models. From the nearlyisothermal models we know that even the migration direction is very sensitive to temperature variations, and we expect that because for our nominal density cooling is not efficient we should 


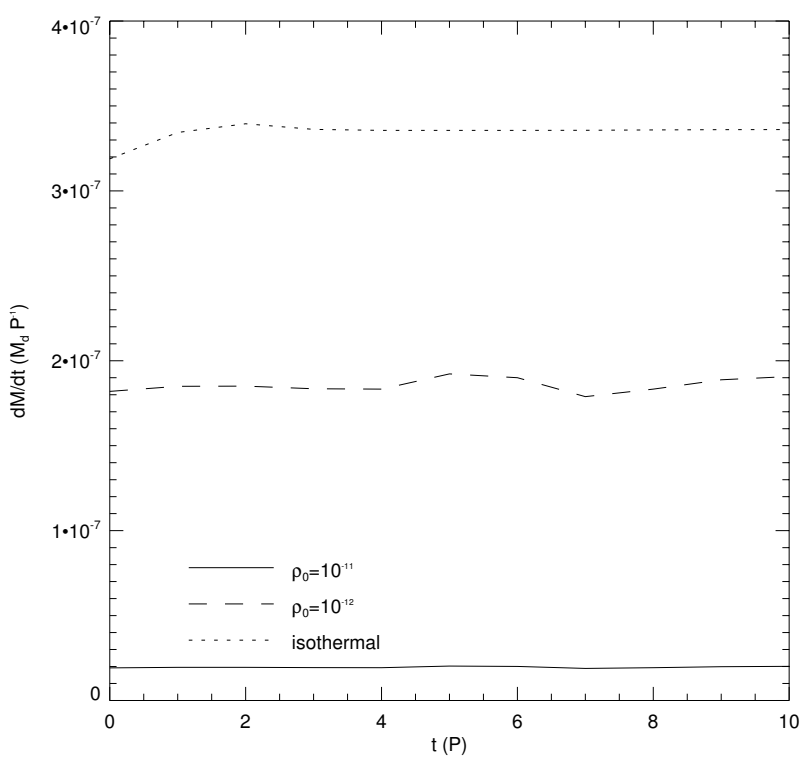

Fig. 16. Accretion rates onto a $5 M_{\oplus}$ planet for radiationhydrodynamical models with two different densities. For comparison, the isothermal accretion rate is also shown.

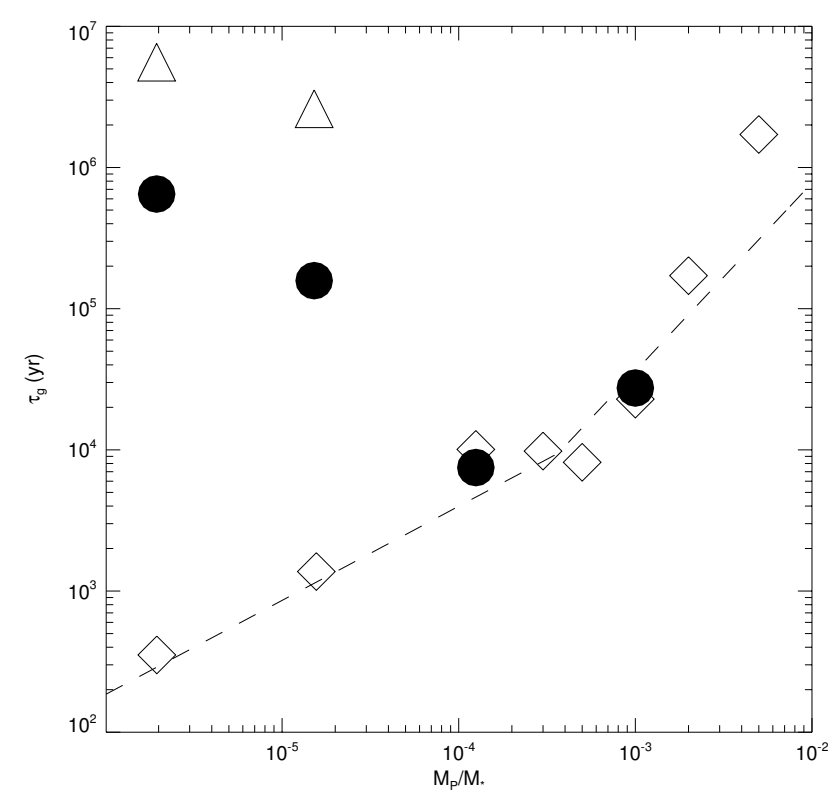

Fig. 17. Same as the right panel of Fig. 6, with the radiationhydrodynamical results (with our nominal initial density of $\rho_{0}=$ $10^{-11} \mathrm{~g} \mathrm{~cm}^{-3}$ ) included as open triangles.

recover at least some of the features of the nearly-isothermal models.

In Fig. 18 we show the total torque acting on the planet as a function of time, for three different radiation-hydrodynamical models. All torques reach a constant value within 10 dynamical time scales, even though the cooling time for the high-density case is somewhat longer. We checked that the torques did not change over several cooling times. Again, as was the case for the nearly-isothermal models, the torque needs some more time to reach a steady value than in the isothermal case.

For our nominal, non-accreting model we observe that the total torque is again positive, indicating outward migration. Its magnitude is comparable to the ordinary type I torque as calculated by Tanaka et al. (2002), and this is much lower than the

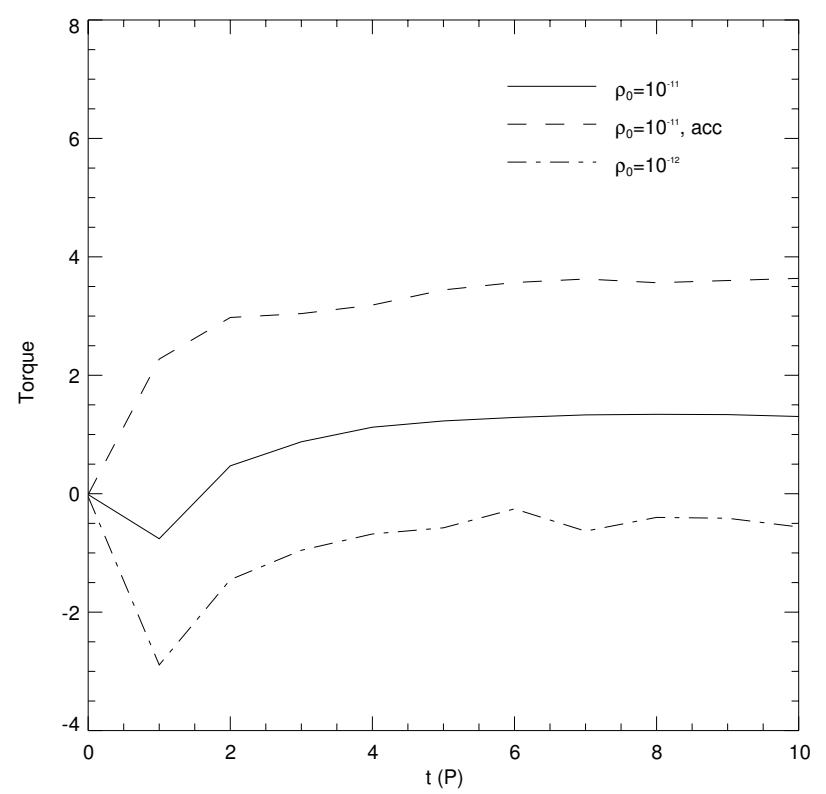

Fig. 18. Total torque onto a $5 M_{\oplus}$ planet for three different radiation hydrodynamical models. The torques are normalized to the absolute value of the analytical result of Tanaka et al. (2002).

magnitude of the torque in the accreting, nearly isothermal models. It turns out that this is due to the accretion flow. Indeed, for our accreting radiation-hydrodynamical model (dashed line in Fig. 18) the magnitude of the torque increases by a factor of 3 compared to the non-accreting case. It is the asymmetric, hot outflow that is responsible for a large part of the positive torque in the accreting model. Radiative heat diffusion tends to lower the magnitude of the torque, because the flow is spread out more evenly around the planet. This effect is not strong, however.

When we lower the density the magnitude of the torque decreases until at some point it becomes negative. This is because the cooling time decreases with decreasing density, and therefore the disk is more and more able to cool towards its initial temperature. From Fig. 18 we see that for a 10 times lower density the total torque is negative, with a magnitude that is approximately a factor 2 lower than the analytical type I torque from Tanaka et al. (2002). As we reduce the density even more the torque approaches the analytical result even more, but the scatter around the equilibrium value increases. This is due to the fact that for such densities the disk becomes very optically thin, which makes the problem less well suited to treat in the diffusion approximation.

For accreting models, we do not reach the type I result, however. The results on accretion indicated already that even for very low densities there is still a very hot bubble formed around the planet, and the subsequent hot outflow comes to dominate the torque, which we found to remain positive even for the case of a 10 times lower density. This is again an indication that accretion and migration of embedded planets are closely linked. In order to make progress, the detailed process of accretion requires further study.

Nevertheless, even for our non-accreting model we find a torque that is positive, which means that the hot accretion flow is not the whole story. It is interesting to see where in the disk this positive torque arises. In Fig. 19 we show the torque on the planet as a function of radius. The low-density case (dash-dotted line), giving rise to a negative torque in Fig. 18, agrees very well with the isothermal model (see Fig. 12). For our nominal density 


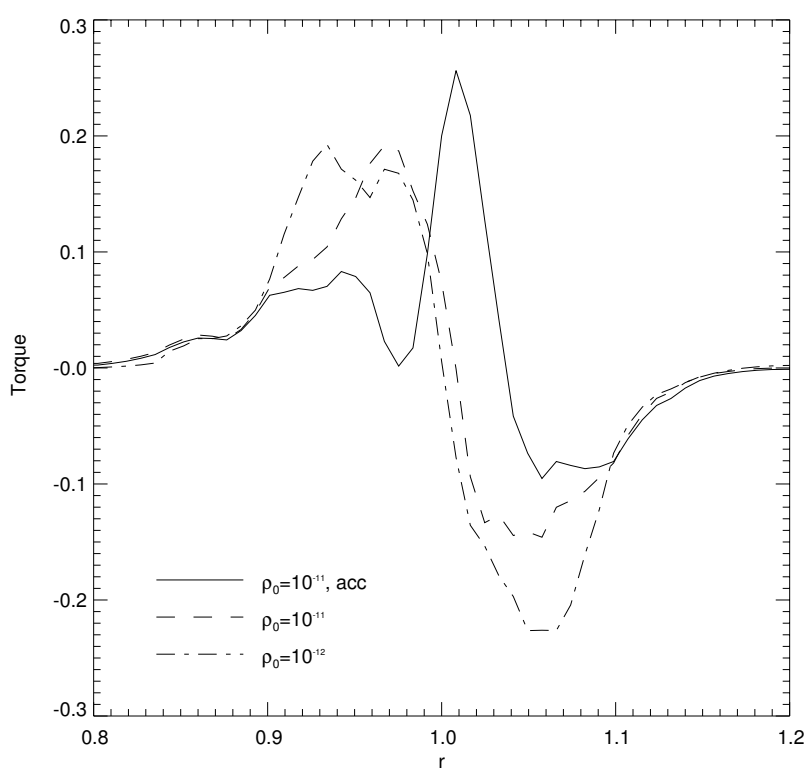

Fig. 19. Radial torque profile for a $5 M_{\oplus}$ planet for three different radiation-hydrodynamical models. The torques are normalized in such a way that the total torque for an isothermal equation of state is equal to -1 .

(indicated by the dashed line in Fig. 19) we see that the magnitudes of the torques due to the inner and the outer spiral wave are lower than for the low-density case. This is due to compressional heating in the waves, as can be seen in the top left panel of Fig. 14. Because of the higher temperature, the same pressure distribution as in the isothermal case requires the density to be lower in the waves, and therefore the torque is less strong.

However, it turns out that the total torque due to the spiral waves is still negative. This means that it is the corotation region that determines the sign of the torque and therefore the direction of migration. In the next section, we will further specify where and how this torque arises using local, adiabatic simulations.

Before we do that, we take a brief look at the accreting radiation-hydrodynamical model in Fig. 19. The whole numerical set-up was the same as for our nominal density model in Fig. 19, only now accretion was turned on. All differences between the solid line and the dashed line in Fig. 19 arise from the accretion process. Note that the sphere of influence of the accretion flow has a radius of approximately $0.1 r_{\mathrm{p}}$, or $2 H$. The outflow of energy released by accretion heats the spiral waves even more, and consequently the torques decrease in magnitude. On top of that, the asymmetric hot bubble seen in Fig. 15 itself exerts a large positive torque onto the planet, which can be identified as the large peak in the torque around $r=1$ in Fig. 19. This peak is less strong than in the nearly-isothermal case (see Fig. 12) because the heat generated by accretion is diffused by radiation over a much larger volume.

\subsection{Local, adiabatic models}

In this section we take a step back and look at local, adiabatic simulations. That is, we keep $\Gamma=1.4$ but we switch off the radiative transport, and we further reduce the computational effort by simulating only a small part of the disk of 5 initial pressure scale heights around the planet. This volume is large enough to capture the basic features of the flow, because the effects of the low-mass planets we consider on the disk remain fairly local. We made sure that the global resolution in these local models matches the highest local resolution of the previously described models.

In these simplified simulations the gas is still heated by compression and gravitational effects, while radiative cooling is absent. This way, these simulations would apply to a very dense region where the cooling time scale is much larger than the dynamical time scale everywhere, also in the upper layers of the disk. Although unrealistic for real protoplanetary disks, these simulations allow us to pin down where the positive torque in a non-accreting model arises while not being hindered by the cooling effects of radiation. These cooling effects will partially wash out the effects of compression, and while the total torque is still positive, its origin is much harder to find.

In Paardekooper \& Mellema (2006a), it was found that in these models, a warm, underdense plume forms behind the planet, which is responsible for the torque reversal. We provide some additional discussion here. Further simulations have indicated that the torque due to temperature effects,

$\mathcal{T}_{\text {temp }}=\int_{\text {disk }} \frac{\mathrm{G} M_{\mathrm{p}} \partial \rho}{\left|\boldsymbol{r}-\boldsymbol{r}_{\mathrm{p}}\right|^{3}} \boldsymbol{r}_{\mathbf{p}} \times\left(\boldsymbol{r}-\boldsymbol{r}_{\mathrm{p}}\right) \mathrm{d}^{3} r$,

is proportional to the radial entropy gradient of the unperturbed disk:

$\mathcal{T}_{\text {temp }} \propto-\mathcal{S} \equiv-\frac{r}{S} \frac{\mathrm{d} S}{\mathrm{~d} r}=-\frac{1}{T} \frac{\mathrm{d} T}{\mathrm{~d} r}+(\Gamma-1) \frac{1}{\rho} \frac{\mathrm{d} \rho}{\mathrm{d} r}$.

In our model disk, $\mathcal{S}$ is negative, which gives a positive torque on the planet. Reversing the entropy gradient, one finds that the torque also changes sign. This is also seen using a different method (Baruteau \& Masset 2007). We leave a detailed discussion of this effect, in particular its emergence from linear theory, to a future publication (Paardekooper \& Papaloizou 2007). Here, we just note that the mechanism depends on the conservation of entropy along streamlines in the horseshoe region, which, in the presence of a background radial entropy gradient, leads to a strong corotation torque. In a barotropic disk, a similar mechanism operates involving the specific vorticity (see Ward 1991). It is interesting to note that, in contrast to the accretion rates, the torque displays discontinuous behavior at $\Gamma=1$. For an adiabatic disk, where density and temperature decrease outward, according to Eq. (18) the entropy gradient reaches a minimum for $\Gamma \rightarrow 1$, leading to a strong positive corotation torque. For $\Gamma=1$, however, we have a locally isothermal disk in which entropy is not conserved along streamlines, which kills the entropy-related corotation torque.

\section{Discussion}

Our results on gas accretion onto low-mass protoplanets suggest that mass flow into the Hill sphere depends critically on the temperature profile of the planetary envelope. In isothermal simulations, pressure gradients correspond to density gradients alone, and therefore a pressure-supported isothermal envelope will have a very high central density. This leads to the relatively high accretion rates in isothermal models. When the energy balance is taken into account, part of the pressure gradient that is needed to sustain the envelope is carried by a gradient in temperature and therefore the corresponding density profile will be less peaked, and therefore the measured accretion rate will go down. Moreover, the energy released in the accretion process raises the central temperature even further, slowing down the mass flow even more. This way, accretion is a self-limiting process, and the 
subsequent accretion rate is more than an order of magnitude below the isothermal result. The values we find are comparable to the solid accretion rates found by Pollack et al. (1996), depending on the mass of the planet. Heating due to this planetesimal bombardment should lead to a similar drop in the accretion rate as observed here. We conclude that accretion rates obtained from isothermal simulations strongly overestimate the growth rate of embedded planets.

Because the release of accretion energy plays such a significant role in accretion, migration and observations of protoplanetary envelopes (Klahr \& Kley 2006) it is important to consider the validity of this accretion recipe. Although at first sight it seems a good idea to conserve total energy and momentum during accretion, it is not entirely clear if this is indeed the case. The momentum carried by the accreted matter, for example, might be transferred to the planet itself, which may induce rotation of the protoplanetary core.

As an example for an alternative to energy-conserving accretion, consider an accretion recipe that conserves entropy. Because at constant entropy the pressure is related to the density as:

$p=K \rho^{\Gamma}$,

we can, using the ideal gas law, immediately write down that

$T=C \rho^{\Gamma-1}$,

for some constant $C$. This means that taking away mass from the grid actually cools the accretion region. In this case the assumption is that the accretion energy is transferred to the planetary core. Note that in using an isothermal equation of state, taking away mass from the grid amounts to conservation of entropy as well. Because accretion plays such a major role in nonisothermal planet-disk interaction, obviously in the growth of the planet but also in the migration behavior, more work on the details of the accretion process is necessary.

But even for non-accreting planet, a hydrostatic envelope is never reached (see also D'Angelo et al. 2003b). Mass constantly flows in and out of the Roche lobe, and it is not yet clear what role these outflows play in planet-disk interaction. In our simulations we have found that the outflow is predominantly equatorial, irrespective of whether the planet is accreting mass or not. The depth of the potential well does influence the outflow, however, because it is driven by the high pressure region at the location of the planet. We found that this outflow did not alter the torque balance in the isothermal limit, but because the gas expands rapidly inside the outflow it affects the temperature in the midplane and it may therefore be of importance in non-isothermal disks.

The most interesting result from this study is the observed reversal of migration direction with respect to isothermal disks. We have tried to isolate the origin of the positive torque arising in adiabatic simulations, and we have found that gas heating in the region where the horseshoe orbits make their U-turn is the dominant effect. This heating is driven by a radial entropy gradient, where a negative gradient results in a positive torque on the planet.

Classical corotation torques tend to saturate when the viscous diffusion time scale across the librating region is longer than the libration time scale (Ogilvie \& Lubow 2003). In our inviscid models, saturation should occur, but a much larger time range is needed to see the effect. The computational costs of radiation-hydrodynamical simulations are too large to achieve this, however. Global adiabatic models may provide more insight in this matter. The new torques arising from a radial entropy gradient may be kept unsaturated by energy transport by radiation, but only if the disk in radiative equilibrium has indeed a radial entropy gradient. More detailed disk models are needed to answer this question.

In our models where we solve the energy equation we have focused on a $5 M_{\oplus}$ planet. One run involved a $0.6 M_{\oplus}$ accreting planet that also moved outward, but more study is necessary to establish the trend, in particular whether it is a purely linear effect.

When the planet gets massive enough to substantially change the density in its direct environment, the situation changes. Due to the lower density near the orbit of the planet the positive corotation torques decrease in strength because the cooling efficiency increases. Note that the cooling time is proportional to the density squared as long as the disk remains optically thick. Therefore the outward migration induced by corotational heating will quickly lose strength when the planet opens up a gap. For gap-opening planets, this mechanism does not operate because the disk near the planet can cool efficiently (density reductions of two orders of magnitude were measured in 2D simulations).

This new migration behavior of low-mass planets raises a few interesting issues. First of all, because the direction of migration depends on the density, there exists a certain radius $R_{0}$ with density $\rho\left(R_{0}\right)$ where the total torque is zero. This radius is the same for all low-mass planets, because it depends on cooling properties of the disk only. Inside $R_{0}$, cooling is not efficient and planets move outward. Outside $R_{0}$, planets move inward through ordinary type I migration. Therefore all low-mass planets near $R_{0}$ in the disk will slowly approach $R_{0}$. In a simple power-law disk there is only one $R_{0}$, which, in the Minimum Mass Solar Nebula is located around 10-15 AU. When the density structure becomes more complicated (for example due to the presence of a gap-opening planet) there may be several locations in the disk where the total torque is zero.

As the low-mass planets approach $R_{0}$, planet-planet interactions will eventually come to dominate. When this happens depends on the magnitude of the torque in that region. Interesting effects were observed for differentially migrating low-mass planets by Cresswell \& Nelson (2006). The planets may lock into mean-motion resonances or be scattered out of the system.

As the disk evolves it slowly loses mass. Therefore, in a simple power-law disk, $R_{0}$ will shift inwards slowly. Once a planet has reached $R_{0}$ either by outward or inward migration, it will slowly migrate inward due to the dispersal of the disk. Therefore the migration time scale for low-mass planets becomes directly coupled to the disk life time. While in the isothermal type I migration scenario migration may proceed on any time scale depending on the disk mass, when the energy budget is taken into account this is no longer the case. Low-mass planet migration will proceed at time scales comparable to the disk life time, which is equal to the viscous time scale of type II migration. Therefore, this scenario of corotational heating provides a solution to the problem of fast-migrating cores.

In using Eq. (1) in calculating the cooling time we have assumed that the planet resides beyond the snow line. Interesting things may happen in regions where the opacity suddenly changes, as was pointed out by Menou \& Goodman (2004). Also, grain growth affects the opacity to a great extent. A disk in which all particles have grown to $\mathrm{cm}$-size will be optically thin, and therefore the mechanism of corotational heating will not apply. However, such efficient growth of particles is not consistent with observations of protoplanetary disks (Dullemond $\&$ Dominik 2005) and therefore some sort of replenishment of small grains is needed. 
Another important effect that will have to be included is irradiation from the central star. Although the disk is not severely perturbed by the low-mass planets considered in this paper, it is desirable to treat the heating of the disk in a self-consistent way. However, detailed models including irradiation from the central source are computationally very expensive and therefore hard to implement in a dynamical model. Flux-limited diffusion is not capable of handling possible shadowing effects properly, and therefore one has to switch to a ray-tracing method.

This new migration behavior should in the end be included in population synthesis models of planet formation (Ida \& Lin 2004a,b, 2005). At the moment, these models require type I migration to be reduced by a factor of a few in order to match the observed extrasolar planet population. The new insights in lowmass planet migration presented in this paper can increase the level of consistency in these models.

\section{Summary and conclusions}

In this paper we have presented the first global radiationhydrodynamical simulations of the interaction between lowmass planets and protoplanetary disks. Specifically, we looked at accretion and migration rates in the mass regime of linear disk response.

We have found that accretion and migration rates depend sensitively on the ability of the disk to radiate away energy generated by compression in the various flow regions around the planet. Compression deep within the planetary envelope leads to a decrease in accretion rate onto the planet of more than an order of magnitude. Compression within the tidal waves generated by the planet leads to a decreased Lindblad torque, which makes the planet move inward but at a slower rate than predicted by analytical models for isothermal type I migration. Compression in the horseshoe region, finally, leads to a large positive corotation torque that is able to change the direction of migration, depending on the radial entropy gradient.

All these effects depend critically on the cooling properties of the disk. For a density appropriate for the Minimum Mass Solar Nebula at 5 AU we find outward migration, while a ten times lower density gives rise to inward migration. Lowering the density even more we are able to reproduce the analytical type I torque. This means that inward type I migration is restricted to the outer parts of protoplanetary disks, or to older disks that have a lower density.

Because low-mass planet migration is driven by the decrease of density in the disk, it proceeds on the viscous time scale, just as type II migration for gap-opening planets. This means that corotational heating is able to solve the problem of protoplanetary cores that move inward too fast to form giant planets. All planets within the region where the cooling time scale is much larger than the dynamical time scale will experience outward migration, depending on the local entropy gradient.

Future studies should be aimed at finding the most appropriate recipe to simulate gas accretion onto the planet, and at including irradiation from the central star to calculate the equilibrium disk temperature profile self-consistently.

Acknowledgements. SP thanks Yuri Levin, Mordecai-Marc MacLow, Peter Woitke and John Papaloizou for useful discussions. We thank Willem Vermin for his assistance at the Dutch National Supercomputer, and an anonymous referee for insightful comments that improved the quality of the paper. This work was sponsored by the National Computing Foundation (NCF) for the use of supercomputer facilities, with financial support from the Netherlands Organization for Scientific Research (NWO).

\section{References}

Artymowicz, P. 1993, ApJ, 419, 166

Artymowicz, P. 2004, in Debris Disks and the Formation of Planets, ASP Conf. Ser., 324, 39

Balbus, S. A., \& Hawley, J. F. 1990, BAAS, 22, 1209

Barrett, R., Berry, M., Chan, T. F., et al. 1994, Templates for the Solution of Linear Systems: Building Blocks for Iterative Methods (Philadelphia, PA: SIAM)

Baruteau, C., \& Masset, F. S. 2007, ApJ, in press

Bate, M. R., Lubow, S. H., Ogilvie, G. I., \& Miller, K. A. 2003, MNRAS, 341, 213

Beckwith, S. V. W., \& Sargent, A. I. 1996, Nature, 383, 139

Bell, K. R. \& Lin, D. N. C. 1994, ApJ, 427, 987

Cresswell, P., \& Nelson, R. P. 2006, A\&A, 450, 833

D’Angelo, G., Henning, T., \& Kley, W. 2002, A\&A, 385, 647

D’Angelo, G., Henning, T., \& Kley, W. 2003a, ApJ, 599, 548

D'Angelo, G., Kley, W., \& Henning, T. 2003b, ApJ, 586, 540

D'Angelo, G., Bate, M. R., \& Lubow, S. H. 2005, MNRAS, 358, 316

de Val-Borro, M., Edgar, R. G., Artymowicz, P., et al. 2006, MNRAS, 370, 529

Dullemond, C. P. 2000, A\&A, 361, L17

Dullemond, C. P., \& Dominik, C. 2005, A\&A, 434, 971

Dullemond, C. P., Dominik, C., \& Natta, A. 2001, ApJ, 560, 957

Eulderink, F., \& Mellema, G. 1995, A\&AS, 110, 587

Goldreich, P., \& Sari, R. 2003, ApJ, 585, 1024

Goldreich, P., \& Tremaine, S. 1980, ApJ, 241, 425

Hayes, J. C., \& Norman, M. L. 2003, ApJS, 147, 197

Ida, S., \& Lin, D. N. C. 2004a, ApJ, 604, 388

Ida, S., \& Lin, D. N. C. 2004b, ApJ, 616, 567

Ida, S., \& Lin, D. N. C. 2005, ApJ, 626, 1045

Jang-Condell, H., \& Sasselov, D. D. 2003, ApJ, 593, 1116

Jang-Condell, H., \& Sasselov, D. D. 2004, ApJ, 608, 497

Jang-Condell, H., \& Sasselov, D. D. 2005, ApJ, 619, 1123

Klahr, H., \& Kley, W. 2006, A\&A, 445, 747

Klahr, H. H., \& Bodenheimer, P. 2003, ApJ, 582, 869

Kley, W. 1989, A\&A, 208, 98

Kley, W. 1998, A\&A, 338, L37

Kley, W. 1999, MNRAS, 303, 696

Kley, W., \& Dirksen, G. 2006, A\&A, 447, 369

Levermore, C. D., \& Pomraning, G. C. 1981, ApJ, 248, 321

Lubow, S. H., Seibert, M., \& Artymowicz, P. 1999, ApJ, 526, 1001

Masset, F. S., \& Papaloizou, J. C. B. 2003, ApJ, 588, 494

Masset, F. S., D'Angelo, G., \& Kley, W. 2006, ApJ, 652, 730

Mellema, G., Eulderink, F., \& Icke, V. 1991, A\&A, 252, 718

Menou, K., \& Goodman, J. 2004, ApJ, 606, 520

Morohoshi, K., \& Tanaka, H. 2003, MNRAS, 346, 915

Nelson, A. F., \& Benz, W. 2003a, ApJ, 589, 556

Nelson, A. F., \& Benz, W. 2003b, ApJ, 589, 578

Nelson, R. P., \& Papaloizou, J. C. B. 2004, MNRAS, 350, 849

Ogilvie, G. I., \& Lubow, S. H. 2003, ApJ, 587, 398

Paardekooper, S.-J., \& Mellema, G. 2006a, A\&A, 459, L17

Paardekooper, S.-J., \& Mellema, G. 2006b, A\&A, 450, 1203

Paardekooper, S.-J., \& Papaloizou, J. C. B. 2007, A\&A, submitted

Papaloizou, J. C. B., \& Terquem, C. 1999, ApJ, 521, 823

Papaloizou, J. C. B., \& Nelson, R. P. 2003, MNRAS, 339, 983

Papaloizou, J. C. B., Nelson, R. P., \& Masset, F. 2001, A\&A, 366, 263

Pollack, J. B., Hubickyj, O., Bodenheimer, P., et al. 1996, Icarus, 124, 62

Roe, P. L. 1981, J. Comp. Phys., 43, 357

Sari, R., \& Goldreich, P. 2004, ApJ, 606, L77

Shakura, N. I., \& Sunyaev, R. A. 1973, A\&A, 24, 337

Stone, J. M., Mihalas, D., \& Norman, M. L. 1992, ApJS, 80, 819

Tanaka, H., Takeuchi, T., \& Ward, W. R. 2002, ApJ, 565, 1257

Terquem, C. E. J. M. L. J. 2003, MNRAS, 341, 1157

Ward, W. R. 1991, in Lunar and Planetary Institute Conference Abstracts, Lunar and Planetary Institute Conference Abstracts, 22, 1463

Ward, W. R. 1997, Icarus, 126, 261 